\title{
Phenotypic, genomic, and transcriptional characterization of Streptococcus pneumoniae interacting with human pharyngeal cells
}

Sheila Z Kimaro Mlacha ${ }^{1,2,7}$, Sandra Romero-Steiner ${ }^{3}$, Julie C Dunning Hotopp², Nikhil Kumar ${ }^{2}$, Nadeeza Ishmael², David R Riley², Umar Farooq², Todd H Creasy², Luke J Tallon², Xinyue Liu², Cynthia S Goldsmith4, Jacquelyn Sampson ${ }^{3}$, George M Carlone ${ }^{3}$, Susan K Hollingshead ${ }^{5}$, J Anthony G Scott ${ }^{1,6}$ and Hervé Tettelin ${ }^{2 *}$

\begin{abstract}
Background: Streptococcus pneumoniae is a leading cause of childhood morbidity and mortality worldwide, despite the availability of effective pneumococcal vaccines. Understanding the molecular interactions between the bacterium and the host will contribute to the control and prevention of pneumococcal disease.

Results: We used a combination of adherence assays, mutagenesis and functional genomics to identify novel factors involved in adherence. By contrasting these processes in two pneumococcal strains, TIGR4 and G54, we showed that adherence and invasion capacities vary markedly by strain. Electron microscopy showed more adherent bacteria in association with membranous pseudopodia in the TIGR4 strain. Operons for cell wall phosphorylcholine incorporation (lic), manganese transport (psa) and phosphate utilization (phn) were up-regulated in both strains on exposure to epithelial cells. Pneumolysin, pili, stress protection genes ( $a d h C-c z c D)$ and genes of the type II fatty acid synthesis pathway were highly expressed in the naturally more invasive strain, TIGR4. Deletion mutagenesis of five gene regions identified as regulated in this study revealed attenuation in adherence. Most strikingly, $\triangle S P \_1922$ which was predicted to contain a B-cell epitope and revealed significant attenuation in adherence, appeared to be expressed as a part of an operon that includes the gene encoding the cytoplasmic pore-forming toxin and vaccine candidate, pneumolysin.
\end{abstract}

Conclusion: This work identifies a list of novel potential pneumococcal adherence determinants.

Keywords: Streptococcus pneumoniae, Gene expression, Microarray, Adherence, Invasion, Genome, Mutagenesis, SP_1922, Ply operon

\section{Background}

Streptococcus pneumoniae (the pneumococcus) is a leading cause of community-acquired pneumonia, meningitis, sepsis and otitis media. It colonizes the human nasopharynx asymptomatically, but can spread to the middle ear and lungs, or penetrate the epithelial cells and enter the bloodstream leading to invasive disease. Worldwide S. pneumoniae is responsible for $>14.5 \mathrm{M}$ cases of invasive disease annually and up to $11 \%$ of all deaths in children $[1,2]$.

\footnotetext{
* Correspondence: tettelin@som.umaryland.edu

${ }^{2}$ Department of Microbiology and Immunology, Institute for Genome Sciences, University of Maryland School of Medicine, 801 W. Baltimore Street, Baltimore, MD 21201, USA

Full list of author information is available at the end of the article
}

Adherence of the pneumococcus to the nasopharyngeal epithelium is essential to successful colonization and constitutes the first step in the invasive route of this pathogen. Previously, pneumococcal adherence and invasion has been studied by gene expression analyses using RT-PCR [3] or microarrays [4-7]. Despite the identification of several key adherence factors, including pneumococcal surface adhesin A (PsaA), pneumococcal serine repeat protein (PsrP), choline binding proteins (CbpA, CbpE), pilus protein (RrgA), plasminogen- and fibronectin-binding proteins ( $\mathrm{PfbA}$ and $\mathrm{PfbB}$ ), pneumococcal adherence and virulence factor $\mathrm{A}$ and $\mathrm{B}$ (PavA and $\mathrm{PavB}$ ) [8-15], our understanding of the molecular mechanisms of pneumococcal adherence and invasion

\section{Biomed Central}


remains incomplete. Our research aims to improve our understanding of these mechanisms.

We measured variation in the adherence of two pneumococcal strains (TIGR4 and G54) to an epithelial cell line from the human pharynx (Detroit 562). We also examined the pathogen-host interaction in each of these two strains with electron microscopy. Next, we studied the genome-wide transcriptional response of $S$. pneumoniae as it adheres to and invades the D562 cells in vitro. We did this by comparing transcription profiles of pneumococcal strains exposed to either D562 cells or simple broth, and by comparing transcription profiles of pneumococci that successfully adhered to D562 cells to those that did not. These studies support the role of known adherence determinants and identified potential novel determinants, some of which have no predicted function. Using deletion mutagenesis, we also demonstrate the possible functional relevance of five of these genes.

\section{Methods}

\section{Pneumococcal strains}

TIGR4, a serotype 4 clinical isolate taken from blood, and G54, a serotype 19F isolate from human sputum, were routinely grown as previously described [8]. Colonies were visualized in a stereoscope with transmitted light as previously described [16] and only strains that were transparent in phenotype were used in this study. Viable counts were performed to determine the exact number of CFU per micro-titer well to be used in each experiment.

\section{In vitro adherence assay}

Detroit 562 (D562) cells, human pharyngeal carcinoma epithelial cells, were purchased from the American Type Culture Collection (CCL-138) and grown and maintained as previously described [8]. Adherence of S. pneumoniae to D562 cells was assessed as previously described [8]. Briefly, D562 cells $\left(10^{5} / \mathrm{ml}\right)$ were seeded in 96-well tissue culture treated plates ( $200 \mu \mathrm{l}$ per well) and grown to confluence. The final cell yield was $1.2 \times 10^{5}$ cells/well after 6 days of incubation resulting in a multiplicity of infection (MOI) of 0.01. Monolayers were washed once with $125 \mu \mathrm{l} /$ well of minimal essential medium with Eagle's salts (EMEM) without L-glutamine and supplemented with 7\% fetal bovine serum (Atlas Biologicals, Fort Collins, $\mathrm{CO})$. To the washed monolayer of each well, $80 \mu \mathrm{l}$ of EMEM was added, followed by $20 \mu \mathrm{l} /$ well of bacterial suspension $\left(10^{3}\right.$ bacteria/well). Plates were incubated for $2 \mathrm{~h}$ at $37^{\circ} \mathrm{C}$ in a $5 \% \mathrm{CO}_{2}$ incubator to allow for adherence then washed $5 \mathrm{X}$ with phosphate-buffered saline (PBS) with $0.2 \%$ bovine serum albumin (BSA) to remove nonadherent pneumococci. A $65 \mu \mathrm{l}$ volume of THYE (Todd-Hewitt broth supplemented with $0.5 \%$ yeast extract), $0.8 \%$ agar and 0.1\% 2,3,5-triphenyl tetrazolium chloride (TTC; Difco Laboratories), was added and the plates were incubated overnight at $37^{\circ} \mathrm{C}$ in a $5 \% \mathrm{CO}_{2}$ incubator. The number of colonies of $S$. pneumoniae adhering to D562 cells was counted using an automated colony counter (AlphaImager; Alpha Innotech, CA) and adherence was expressed as $100 \mathrm{X}$ (CFU of adherent bacteria / CFU of inoculated bacteria) per well. These counts are inclusive of bacteria that were internalized within the cells. Values for 4 replicate wells were averaged and 3 independent experiments were performed. Differences in adherence capacity between TIGR4 and G54 were tested by Student's $t$-test.

For microarray experiments, aliquots $(1 \mathrm{ml})$ of pneumococcal TIGR4 and G54 strains containing $10^{7}$ bacteria were centrifuged, washed in PBS, and resuspended in $15 \mathrm{ml}$ of EMEM. Confluent D562 cells, grown in $175 \mathrm{~cm}^{2}$ cell culture flasks (Corning Costar Co., MA, USA), were inoculated with these strains and incubated for $2 \mathrm{~h}$ at $37^{\circ} \mathrm{C}$ in a $5 \% \mathrm{CO}_{2}$ incubator. Nonadherent bacteria (contained in the spent cell culture medium) were removed and collected by differential centrifugation to remove eukaryotic cellular debris. Epithelial cells were washed $3 \mathrm{X}$ with PBS and then treated immediately with $10 \mathrm{ml}$ of RNAprotect (Qiagen, Germany). For experiments with G54, adherent bacteria were dissociated from host cells by treatment with trypsin-EDTA; for TIGR4, which adhered and invaded to D562 cells extremely efficiently, dissociation was achieved by lysis with $0.1 \%(\mathrm{w} / \mathrm{v})$ saponin in PBS followed by sonication using $5 \mathrm{~s}$ pulses for $1 \mathrm{~min}$. Bacteria were subsequently harvested by differential centrifugation (1st step: $800 \mathrm{x} \mathrm{g}$ for $5 \mathrm{~min}$ to remove mammalian cells, 2nd step: $4400 \mathrm{rpm}$ for $10 \mathrm{~min}$ ). Control bacteria, which were not exposed to host cells, were suspended in EMEM medium and then prepared in parallel and treated identically to adherent and nonadherent bacteria. They were also treated with $10 \mathrm{ml}$ of RNAprotect to minimize RNA metabolism and degradation. Pellets were stored at $-80^{\circ} \mathrm{C}$.

The difference in treatments between G54 and TIGR4 stems from the chronology of the experiments. We began by testing the adherence and expression of the G54 strain. Then we attempted to apply the same method to recover the adherent population of the TIGR4 strain, but we were unable to retrieve enough TIGR4 bacteria for extraction of sufficient amounts of RNA. A significant proportion of bacteria remained lodged in the pharyngeal cells (as later evidenced by the electron microscopy results). Liberation of TIGR4 pneumococci therefore required selective lysis of eukaryotic cells by saponin treatment and sonication. For various reasons, we did not go back and repeat all G54 experiments, however it should be noted that prior to any 
detachment treatment all bacteria were incubated with $10 \mathrm{ml}$ of RNAprotect (Qiagen) for immediate stabilization of RNA and to minimize RNA degradation. This incubation should prevent any change in bacterial RNA profiles during further treatments.

\section{Invasion assay}

Internalization of bacteria into D562 cells was assessed by a gentamicin assay as previously described [17]. Briefly, confluent D562 cells were inoculated with $S$. pneumoniae as described in the adherence assay. After $1 \mathrm{~h}$ and $45 \mathrm{~min}$ incubation, the cells were treated with $200 \mu \mathrm{g} / \mathrm{ml}$ of gentamicin for $15 \mathrm{~min}$ to kill extracellular bacteria. Monolayers were washed 5 times with PBS with $0.2 \%$ BSA to remove non-adherent pneumococci and any residual gentamicin. As in the adherence assay, monolayers were overlaid with THYE agar with $1 \%$ TTC and incubated overnight at $37^{\circ} \mathrm{C}, 5 \% \mathrm{CO}_{2}$. Internalized bacteria (CFU) were quantified using an automated colony counter (AlphaImager; Alpha Innotech, CA). Differences in invasion capacity between TIGR4 and G54 were tested by Student's $t$-test.

Pneumococcal strains TIGR4 and G54 strains were tested for gentamicin sensitivity using gentamicin discs of $10 \mu \mathrm{g}$ (Oxoid Ltd., Hampshire, England). Both strains had comparable gentamicin inhibition zone diameters of $10.3 \mathrm{~mm}$ for TIGR4 and $10 \mathrm{~mm}$ for G54. The time and concentration of antibiotics used in these experiments were optimized for the D562 cell line. Preliminary timecourse experiments by Rajam et al. [17] (data not shown) showed that a $200 \mu \mathrm{g} / \mathrm{ml}$ concentration of gentamicin was sufficient to kill extracellular pneumococci in 15 minutes. Longer time-points resulted in gentamicin penetrating the Detroit 562 cells, which resulted in internal killing rather than extracellular killing.

\section{Electron microscopy}

D562 cells, grown to confluence in $175 \mathrm{~cm}^{2}$ cell culture flasks (Corning Costar Co., MA), were inoculated as described above with $10^{7}$ bacteria (MOI of 10). After a 2-h incubation step, monolayers were washed with EM buffer (100 mM sodium phosphate, $\mathrm{pH} 7.6,3 \mathrm{mM} \mathrm{KCL}$, $3 \mathrm{mM} \mathrm{MgCl}_{2}$ ) and gently layered with $2.5 \%$ buffered glutaraldehyde $(3 \mathrm{ml})$. The cells were scraped with a cell scraper (Corning Inc., Corning, NY) within $1 \mathrm{~min}$ and the cell suspension was centrifuged at $800 \mathrm{x} \mathrm{g}$ for $10 \mathrm{~min}$ at $24^{\circ} \mathrm{C}$. The cell pellets were then incubated for $1 \mathrm{~h}$ at $4^{\circ} \mathrm{C}$ to fix them. Pellets were washed once with EM buffer and stored at $4^{\circ} \mathrm{C}$. D562 cell specimens were embedded in a mixture of Eponate 12 and Araldite 502 [18] and thin sections of plastic-embedded cells were examined by electron microscopy.

\section{RNA preparation}

Total RNA was isolated from three populations of RNA stabilized bacteria: adherent, non-adherent and bacteria growing freely in EMEM. Total RNA was isolated using TRIzol (Invitrogen Life Technologies, USA) in a lysing matrix containing silica beads and a FastPrep Instrument (Qbiogene, Inc, CA). Purification was done using i) phase separation with TRIzol and ii) column exclusion with the RNeasy Mini Kit (Qiagen, Germany) according to the manufacturers instructions. Contaminating genomic DNA was removed by DNase treatment. RNA concentration was determined using the NanoDrop ND-1000 spectrophotometer (NanoDrop Technologies, USA) and the integrity was assessed using the prokaryote and eukaryote total RNA chips on the Agilent 2100 Bioanalyzer (Agilent Technologies, Germany). Good quality RNAs were amplified using Genisphere's SenseAMP kit (Genisphere Inc., PA) to increase the yield of RNA for microarray experiments.

\section{Probe preparation and microarray hybridization}

The S. pneumoniae microarrays used in this study consisted of 3482 70-mer oligonucleotide probes from the genomes of 3 pneumococcal strains (TIGR4, R6 and G54) as well as 10 amplicons and 500 oligonucleotides (70-mers) from Arabidopsis thaliana which served as negative controls. All probes were aligned against the HG19 version of the human genome using BlastN and no significant hits were obtained. Probes were printed $5 \mathrm{X}$ on aminosilane-coated slides (SCHOTT Nexterion). The microarrays (version 6) were kindly provided by the Pathogen Functional Genomics Resource Center (PFGRC) at the J. Craig Venter Institute and experiments were performed as previously described [19]. Briefly, $2 \mu \mathrm{g}$ of the total RNAs to be compared were reverse transcribed into single-stranded cDNA using 200 U Superscript II reverse transcriptase (Invitrogen), $6 \mu \mathrm{g}$ random hexamers (Invitrogen), 1X first strand buffer (Invitrogen), $10 \mathrm{mM}$ dithiothreitol (DTT), $0.5 \mathrm{mM}$ dATP, $0.5 \mathrm{mM}$ dCTP, $0.5 \mathrm{mM}$ dGTP, $0.3 \mathrm{mM}$ dTTP and $0.2 \mathrm{mM}$ of aminoallyl-modified nucleotide (Invitrogen). The mixture was incubated overnight at $42^{\circ} \mathrm{C}$ and the reaction stopped by addition of $10 \mu \mathrm{l} 0.5 \mathrm{M}$ EDTA and $1 \mathrm{M} \mathrm{NaOH}$. Amine-modified cDNA was purified using QIAquick PCR purification kit (Qiagen, Germany) followed by chemical labeling with Cy3- or Cy5-NHS-ester fluorescent dyes (Amersham-Pharmacia, Piscataway, NJ) in a final step. Slides were pre-hybridized in a $50 \mathrm{ml}$ solution of $5 \mathrm{X}$ SSC, $0.1 \%$ SDS and $1 \%$ BSA for $30 \mathrm{~min}$ at $42^{\circ} \mathrm{C}$, washed $4 \mathrm{X}$ in water and once in isopropanol, then dried by brief centrifugation. Labeled probes were re-suspended in hybridization buffer (30\% formamide, 5X SSC, 0.1\% SDS, $0.6 \mu \mathrm{g} / \mu \mathrm{L}$ salmon sperm DNA) and hybridized to the microarray slides in a $42^{\circ} \mathrm{C}$ water bath for $16-20 \mathrm{~h}$. Slides were washed twice in a low stringency buffer (2X SSC, 
$0.1 \% \mathrm{SDS}$ ) at $55^{\circ} \mathrm{C}$ for $5 \mathrm{~min}$, twice in a medium stringency buffer $(0.1 \mathrm{X} \mathrm{SSC,} 0.1 \% \mathrm{SDS})$ at room temperature for $5 \mathrm{~min}$ and finally twice in a high stringency buffer (0.1X SSC) at room temperature for $5 \mathrm{~min}$, and then dried by brief centrifugation. Synthesized cDNA from each RNA sample from three independent cell cultures was hybridized on three separate microarray slides (biological replicates), and independently synthesized cDNA from each of these same RNA samples was hybridized in a repeat dye-swap experiment (technical replicates) to test technical reproducibility. Additional file 1 summarizes the experimental design.

\section{Data collection, normalization, and analysis}

Dried slides were scanned using a GenePix 4000B dualcolor laser scanner (Axon Instruments, CA, USA) and saved as two independent 16-bit TIFF files corresponding to the two labeled probes (Cy3 and Cy5). Data were analyzed using the TM4 microarray software suite [20]. Spot intensities were quantified using Spotfinder v3.1.1. The two channels were normalized using the iterative log mean centering algorithm implemented in the MIDAS software (v2.19) and the fluorescence ratios were calculated from the normalized values. Genes with significant changes in expression were identified using the significance analysis of microarrays (SAM) test implemented in the MeV software (v4.2) with $\Delta=1.18$ for TIGR4 and $\Delta=$ 1.98 for G54 ( $\Delta$ cutoff corresponded to a false positive rate of $0 \%$ ). Only genes that had $\geq 15$ individual high quality data points were included in the analysis. A cutoff of mean fold change of $\geq 2$ was used. This gene list was supplemented with genes that did not meet the fold threshold value but appeared to be co-regulated as a part of an operon. Up and down-regulated genes were classified into meaningful functional categories using EASE (MeV). EASE statistically calculates the probability that a biological theme (TIGR4 or G54 role categories) is over-represented in the gene list of interest compared to the representation of that theme on the microarray. In our study, $p$-values of $<0.001$ were considered significant (EASE, Fisher's exact test with Bonferroni step down correction). Significance was determined for each strain separately.

SAM two-class paired analysis (analogous to a between subjects $t$-test) was used to identify genes exhibiting statistically significant differences in gene expression between adherent/medium-treated control and non-adherent /medium-treated control. A ratio of these gene expression ratios (referred to as ratio index) was calculated for these significantly regulated genes and an arbitrary threshold of 1.5 fold change was set on the ratio index.

\section{Validation of microarray data using real-time qRT-PCR} Reverse transcription was carried out using the QuantiTect Reverse Transcription Kit (Qiagen, Germany) in accordance with the manufacturer's instructions. Briefly, $1 \mu \mathrm{g}$ of total RNA was incubated in genomic DNA (gDNA) Wipeout Buffer (7X) and RNase-free water and incubated at $42^{\circ} \mathrm{C}$ for $2 \mathrm{~min}$ to remove contaminating gDNA. The cDNA was synthesized from the RNA using Quantiscript reverse transcriptase, Quantiscript RT buffer (5X) and a primer mix consisting of long random primers and oligo-dT. The reaction was incubated at $42^{\circ} \mathrm{C}$ for $15 \mathrm{~min}$ and then at $95^{\circ} \mathrm{C}$ for $3 \mathrm{~min}$ to inactivate the Quantiscript RT. Quantitative real-time PCR was performed as previously described [19]. Dilutions of the cDNA $(0.25 \mu \mathrm{l}$ of stock cDNA per $20 \mu \mathrm{l}$ reaction) were used as template in a reaction containing $2 \mathrm{X}$ QuantiTect SYBR Green mix (Qiagen, Germany), RNase free water and gene-specific primers (Additional file 2). The qRTPCR was conducted using an ABI7900HT machine (Applied Biosystems). The reactions were denatured at $95^{\circ} \mathrm{C}$ for $15 \mathrm{~min}$ followed by amplification with 45 cycles of $94^{\circ} \mathrm{C}$ for $15 \mathrm{~s}, 55^{\circ} \mathrm{C}$ for $30 \mathrm{~s}$ and $72^{\circ} \mathrm{C}$ for $30 \mathrm{~s}$. The qRT-PCR data was analyzed using a comparative cycle threshold $(\Delta \mathrm{Ct})$ method [21]. The average $\mathrm{Ct}$ values of a test sample were compared to that of a control sample (e.g. $\left.\mathrm{Ct}_{\text {TIGR4 control }}-\mathrm{Ct}_{\text {TIGR4 adherent }}\right)$. The $\Delta \mathrm{Ct}$ was normalized to genes that did not exhibit any significant change in expression as identified by the microarray experiments: an average of SP_0002 (DNA polymerase), SP_0085 (ribosomal protein) and SP_2135 (ribosomal protein). Each sample of each biological replicate was tested three times.

\section{RNA Sequencing}

The transcriptome of S. pneumoniae TIGR4 adhering to D562 host cells was determined using RNA-Seq on the Illumina Genome Analyzer platform as per the manufacturer's instructions. Briefly, $10 \mu \mathrm{g}$ of total RNA was partially depleted of rRNA using the MicrobeExpress kit (Ambion) then fragmented in a buffer with divalent cations at $94^{\circ} \mathrm{C}$ for $5 \mathrm{~min}$. Double-stranded cDNA was synthesized from the fragmented RNA using SuperScript II (Invitrogen) and random primers. The fragment ends were repaired using T4 DNA polymerase and the DNA polymerase Klenow fragment in the presence dNTPs to remove $3^{\prime}$ overhangs and fill in $5^{\prime}$ overhangs. Subsequently, a 3' "A" overhang was added using a DNA polymerase Klenow fragment lacking $3^{\prime}$ to $5^{\prime}$ exonuclease activity in the presence of dATP. Illumina adaptors with a single 3' " $\mathrm{T}$ " overhang were ligated to both ends of the DNA fragments. cDNA of the appropriate size range was gel purified and amplified, and Illumina sequencing conducted with unpaired reads 36 nt in length. $13,404,147$ high quality sequencing reads were obtained and $95 \%$ of them mapped to the reference $S$. pneumoniae TIGR4 complete genome sequence using the ELAND alignment algorithm (Illumina). The reads 
and alignment data were deposited at the NCBI Gene Expression Omnibus (GEO) database under accession number: GE44947.

\section{Construction of knockout mutants}

In selecting genes for knockout experiments, the features we sought were one or more of the following: (i) significant up-regulation on contact with epithelial cells in the microarray experiments; (ii) up-regulation in the more adherent strain (TIGR4); (iii) ratio index (adherent/ control) / (non-adherent/control) $\geq 1.5$ in both strains; (iv) conservation among the sequenced strains of pneumococci with the exception of known regions of diversity; (v) no prior existing experimental evidence of association with pneumococcal adhesion; (vi) large hypothetical proteins; and (vii) plausibility of knocking out a gene that was part of an operon without causing a polar effect. Preparation of mutagenic constructs, restriction digestion, ligation and transformation were performed using the PCR ligation mutagenesis strategy (insertion deletion mutagenesis) previously described [22]. Briefly, two pairs of gene-specific primers, $\mathrm{J} 1 / \mathrm{J} 2$ and J3/J4, were used to amplify the upstream and downstream flanking regions of a number of target genes. J2 and J3 were modified to contain KpnI and SacI restriction sites. A third pair of primers, KanF and rpslR, were used to amplify a $1.3-\mathrm{kb}$ positive/negative selection cassette kan-rpsL $L^{+}$that was previously introduced in S. pneumoniae strain $403 \mathrm{~J}$ [23]. The kan-rpsL $L^{+}$cassette was comprised of a kanamycin resistant marker (kan) and a counter-selectable marker rpsL ${ }^{+}$ which conferred dominant streptomycin sensitivity in a streptomycin resistant pneumococcal strain. All the primers used in this study are listed in Additional file 3. Amplification proceeded for 34 cycles as follows: denaturation at $95^{\circ} \mathrm{C}$ for $30 \mathrm{~s}$, annealing at $62^{\circ} \mathrm{C}$ (or $55^{\circ} \mathrm{C}$ depending on the primers) for $30 \mathrm{~s}$, and elongation at $72^{\circ} \mathrm{C}$ for $1 \mathrm{~min}$. Amplicons were purified, digested overnight at $37^{\circ} \mathrm{C}$ using $\mathrm{KpnI}$ or SacI restriction enzymes and purified again to completely inactivate the enzyme. The 3 digested PCR products were then ligated using $1 \mu \mathrm{g}$ of T4 DNA ligase (Invitrogen, CA, USA) in ligase buffer at room temperature for $2 \mathrm{~h}$.

\section{Transformation}

Genetic transformation of the ligation products into $S$. pneumoniae strain TIGR4-Z5 (transparent phenotype of a TIGR4 strain) was performed as follows. A series of transformation stocks were prepared by growing $S$. pneumoniae in competence media (THYE with $0.2 \%$ BSA, $0.2 \%$ glucose, and $0.02 \% \mathrm{CaCl}_{2}$ ) at $37^{\circ} \mathrm{C}$ in a $5 \%$ $\mathrm{CO}_{2}$ incubator to an $\mathrm{OD}_{600 \mathrm{~nm}}$ of 0.4 , and freezing down the cultures in $20 \%$ glycerol. A $1 \mathrm{ml}$ frozen aliquot of transformation stocks was thawed out and grown in competence media to exponential phase $\left(\mathrm{OD}_{600 \mathrm{~nm}}=0.4\right)$ at $37^{\circ}$
$\mathrm{C}$ in a $5 \% \mathrm{CO}_{2}$ incubator. The cells were subsequently diluted in competence medium (1:100) and transformed with $10 \mu \mathrm{l}$ of the ligation products and $500 \mathrm{ng}$ of the competence peptide CSP-2 and incubated for $2 \mathrm{~h}$ at $37^{\circ} \mathrm{C}$. Cells were then plated on blood agar plates with antibiotics and incubated for up to $48 \mathrm{~h}$. S. pneumoniae strain Ali 9.2 containing a kan-rpsL $L^{+}$cassette in place of the ali gene, was used as the positive control and a no-DNA control plate was included to check for background resistance activities in the recipient cells. Transformants were selected based on their resistance to kanamycin $\left(\mathrm{Kn}^{\mathrm{R}}\right.$; positive selection) and susceptibility to streptomycin $\left(\mathrm{Sm}^{\mathrm{S}}\right.$; negative selection). Confirmation of gene replacement was performed using colony PCR. Individual colonies that were $\mathrm{Kn}^{\mathrm{R}}$ and $\mathrm{Sm}^{\mathrm{S}}$ were used as template in a PCR reaction using two pairs of primers (J1/kanR and rpslF/J4). Presence of a PCR product of the expected size was indicative of the presence of the cassette in the mutant strain and at the correct location. Colonies with the correct size were re-grown in THYE broth and a glycerol stock was kept at $-80^{\circ} \mathrm{C}$ for use in adherence assays. The adherence assay to test the ability of mutant strains to bind to D562 cells was similar to that described above.

\section{Hemolytic activity of TIGR4 wild type and $\triangle$ SP1922 isogenic mutant}

S. pneumoniae TIGR4 wild type and $\Delta$ SP_1922 were grown to an $\mathrm{OD}_{600 \mathrm{~nm}}$ of 0.4 in THYE. The cells were centrifuged at $3000 \mathrm{~g}$ for $20 \mathrm{~min}$, and the cell pellets were washed once with PBS and re-suspended in $2 \mathrm{ml}$ of PBS. Bacteria were sonicated for $5 \mathrm{~min}$ at $50 \%$ duty cycle using an ultrasonicator (Branson Sonifier 450, USA) in an ice container. Cell lysates were centrifuged at 20,000 $\times$ g for $30 \mathrm{~min}$ at $4^{\circ} \mathrm{C}$ and the supernatants (cell extract) were transferred to a clean tube. Serial dilutions of the cell extracts in PBS (1 ml volume) were prepared. Human erythrocytes were washed twice in cold PBS and were added to the cell extracts to yield a final concentration of $2 \%(\mathrm{v} / \mathrm{v})$. The suspension was incubated at $37^{\circ} \mathrm{C}$ for $30 \mathrm{~min}$ then centrifuged gently at $800 \mathrm{~g}$ for $10 \mathrm{~min}$. Supernatants were transferred to a clean microtiter plate and the absorbance of released hemoglobin was determined at $540 \mathrm{~nm}$ using a microplate spetrophotometer (Labsystems Multiskan RC, Fisher Scientific, MA). The positive control was saponin, a known hemolytic agent, and the negative control was $0.5 \%$ dimethyl sulfoxide (DMSO). The relative hemolytic activity (\% lysis) at each concentration was determined using a control containing $2 \%(\mathrm{v} / \mathrm{v})$ erythrocytes and deionized water that was considered as $100 \%$ hemolysis. All experiments were carried out in triplicate.

\section{RT-PCR confirmation of the SP_1922 - ply operon}

Total RNA was isolated from $S$. pneumoniae strain TIGR4 grown in THYE using the Qiagen RNeasy Mini 
Kit (Qiagen, Germany) and treated with DNase on column and in solution. Reverse transcription was performed using SuperScript II Reverse Transcriptase (Invitrogen, Carlsbad, CA) following the manufacturer's instructions. Total RNA (800 ng) was incubated with each of 10 gene-specific primers (Additional file 4) at $65^{\circ} \mathrm{C}$ for $5 \mathrm{~min}$ and quick chilled on ice. To this mixture was added 5X First-Strand Buffer and 0.1 M DTT, and the samples were incubated at $42^{\circ} \mathrm{C}$ for $2 \mathrm{~min}$. SuperScript II RT was then added and the samples were incubated at $42^{\circ} \mathrm{C}$ for $50 \mathrm{~min}$, followed by enzyme inactivation by heating to $70^{\circ} \mathrm{C}$ for $15 \mathrm{~min}$. The $10 \mathrm{cDNAs}$ were pooled and PCR was performed with NEB Taq $2 \mathrm{X}$ Master Mix (NEB, Ipswich, MA), following the standard protocol and using combinations of gene-specific primers spanning various regions within the pneumolysin operon (Additional file 4).

\section{Results}

\section{Pneumococcal adhesion to and internalization within} D562 pharyngeal cells

Adhesion of S. pneumoniae to D562 pharyngeal epithelial cell monolayers was assayed in vitro, and invasion was assessed based on gentamicin selection for intracellular bacteria. Two pneumococcal strains were tested, TIGR4 and G54. The mean adherence capacity to D562 cells was significantly higher $(p$ value $<0.05)$ for TIGR4 (545 CFU/well \pm 40 ) than G54 (89 CFU/well \pm 13 ). This translated to $54.5 \%$ of the inoculum for TIGR4 and $8.9 \%$ of the inoculum for G54. The internalization capacity also varied significantly ( $p$ value $<0.05$ ) between the 2 strains; 233 CFU/well \pm 12 for TIGR4 (23.3\% of the inoculum) and $3 \mathrm{CFU} /$ well for G54 (0.3\% of the inoculum). When we analyzed the growth patterns of TIGR4 and G54 during the 2-h assay, we found that some growth occurred in EMEM but the differences were not statistically significant up to $2 \mathrm{~h}$ (Additional file 5). We adjusted the adherence and invasion calculations for growth, resulting in values of $37.2 \%$ and $4.3 \%$ for TIGR4 and G54, respectively. Similarly, invasion was $15.8 \%$ and $0.14 \%$ for TIGR4 and G54, respectively.

These interactions were visualized using electron microscopy. Unlike G54, TIGR4 pneumococci were found in larger numbers adjacent to and within epithelial cells (Figure 1). Pneumococci attached to the monolayer of epithelial cells either as micro-colonies (Figure $1 \mathrm{~A}$ ) or as single cells (Figure $1 \mathrm{~B}, \mathrm{C}, \mathrm{D}$ ), and this was accompanied by the formation of elongations of the epithelial cell out towards and around the bacterial cells. The images indicated that pneumococci were internalized into host cell vacuoles (Figure $1 \mathrm{E}, \mathrm{F}$ ).

Since TIGR4 and G54 differed significantly in their ability to adhere to and invade D562 cells, we sought to determine genetic differences between the strains. Direct BLASTP analysis (e-value cut-off of $10 \mathrm{e}^{-15}$ ) of TIGR4 and G54 proteins predicted from the whole genomes revealed that over $10 \%$ of each strain's proteins are unique with respect to the other strain $(15.7 \%$ TIGR4 and $12.4 \%$ G54). Representation of shared and unique proteins along the genomes revealed a number of strain-
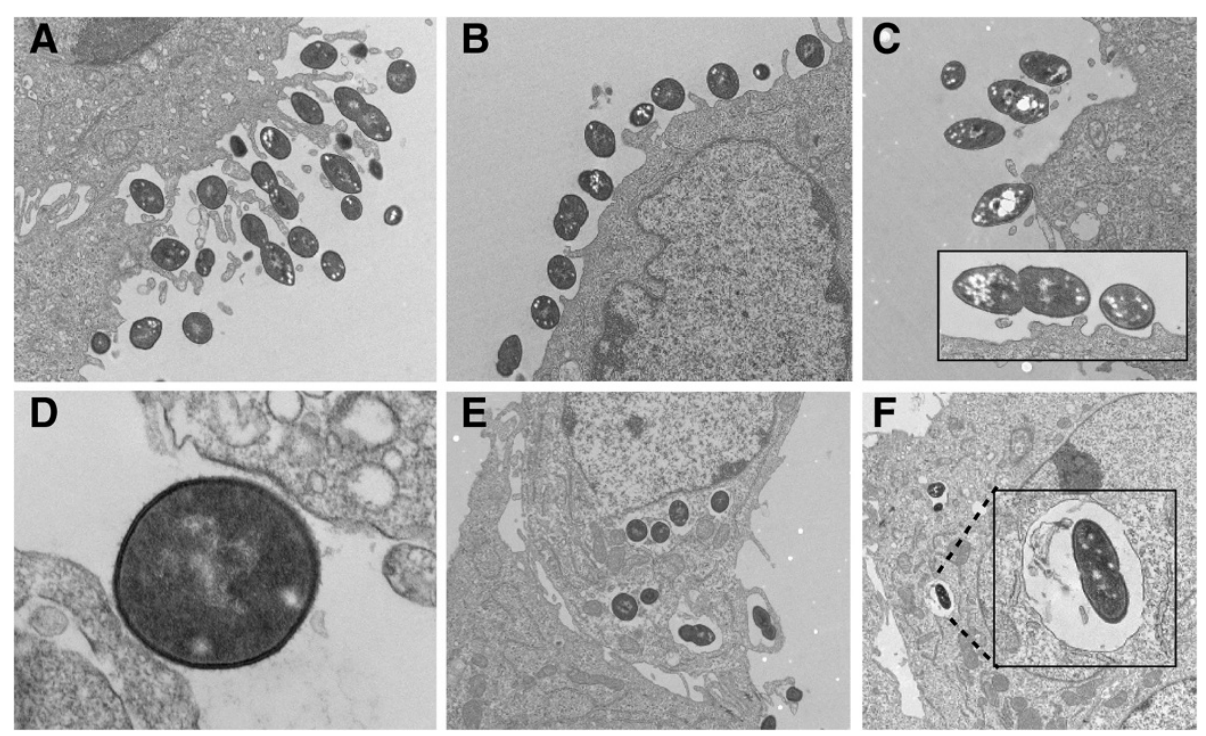

Figure 1 Electron micrographs of D562 epithelial cells incubated with TIGR4 and G54 strains for 2 hours. Pneumococci attach to the host cells either as micro-colonies (A - TIGR4) or single colonies (B, D - TIGR4, C (including inset) - G54) and are surrounded by host pseudopodia. Bacteria invade host cells and are internalized into cytoplasmic vacuoles (E - TIGR4 and F - G54). Original magnifications A, 4400X; B, 4400X; C, 4400X (inset, 15000X); D, 26000X; E, 3200X; F, 1650X (inset, 6500X). 
specific genomic islands encoding proteins potentially involved in the adherence and invasion processes (Figure 2, Additional file 6).

\section{Gene expression analyses}

We analyzed the transcriptome of both pneumococcal strains upon exposure and adherence to human pharyngeal cells. DNA microarrays were used to analyze the gene expression profiles of S. pneumoniae in the following two ways: (i) transcription profiles of bacteria adhering to host cells were compared with those of bacteria grown in tissue culture medium in the absence of host cells (i.e., adherent bacteria vs. culture-medium-treated bacteria), and (ii) transcriptional profiles of adherent bacteria were compared to those of non-adherent bacteria exposed to the host cells (i.e., adherent bacteria vs. nonadherent bacteria). A schematic representation of the experimental setup is shown in Additional file 1.

Analysis of gene expression in adherent bacteria compared to culture medium-treated bacteria: After analysis of statistical significance, application of a 2 -fold cutoff on expression ratios and addition of genes likely to be co-transcribed (see Methods), TIGR4 differentially regulated 154 genes while G54 regulated 242 genes (Additional file 7, Figure 2 - circles 5-8). The regulation ratios varied from 0.12-14.4. Eighty-four genes were commonly regulated by the adherent population of the two strains. Differentially expressed genes were classified into functional categories (Additional file 7). Categories that were significantly over-represented (Figure 3A and 3B) included 'cellular processes', 'cell envelope', 'transport and binding' and 'fatty acid and phospholipid metabolism' $(p<0.001$; Fisher's exact test, Bonferroni step down correction applied).

Comparison of adherent and non-adherent pneumococci incubated in the presence of host cells: We hypothesized that not only do bacteria remodel their transcriptome on sensing the presence of host cells, but that the degree of adjustment varies within the individual bacterial cells in the population eventually determining the outcome - successful attachment to host cells. Since we did not directly hybridize adherent bacteria against non-adherent bacteria on the same array, we compared the ratio of the adherent vs. control against the ratio of non-adherent vs. control (ratio index). This is mathematically equivalent to the ratio of adherent vs. non-adherent. To increase the sensitivity to meaningful functional differences between adherent and nonadherent bacteria as opposed to between adherent and culture medium-treated bacteria, we used an arbitrary cutoff of 1.5 fold change on the ratio index. Using this ratio cutoff and a statistical test of significance, 282 and 287 genes were differentially regulated in TIGR4 and G54, respectively. Of these, 79 were coordinately expressed (directionally) in both strains (Additional file 8). We infer that these commonly regulated genes are the basic set of genes associated with successful functional adherence.

Successful adhesion was associated with increases in the transcription of 412 genes in a strain-specific manner (Additional file 8). A few of the genes that were upregulated in TIGR4 alone correspond to genes identified in the previous section and include: adhesins ( $r l r$ islet and glycosylated proteins), transporters (sodium SP_0737, amino acid SP_1502), fatty acid metabolism genes and several hypothetical proteins. Genes that code for hypothetical proteins were the most highly represented. These results provide a novel approach to the analysis of genes whose expression is important for intimate adherence and/or invasion of pneumococci to epithelial cells.

\section{qRT-PCR confirmation of gene regulation}

A subset of $21 \mathrm{~S}$. pneumoniae genes were selected for qRT-PCR validation of expression levels based on: i) a range of gene expression values i.e., up-, down- and non-regulated and ii) biological interest. The data were analyzed in separate groups for each strain and for adherent and non-adherent phenotypes. Each sample of each biological replicate was analyzed three times. In all four groups there was a strong positive correlation (correlation coefficient $r>0.968$ ) between the microarray results and the qRT-PCR (Figure 4), supporting the validity of the microarray method. The qRT-PCR results from the TIGR4 adherent sample were further analyzed using the RNA-Seq method. Both techniques provide an absolute measure of the transcript abundance, with low abundance transcripts displaying a low average coverage in RNA-Seq and a high cycle threshold (Ct) in qRT-PCR. Additional file 9 shows the expected logarithmic correlation between the Ct observed in qRT-PCR and the average coverage observed in RNA-Seq for each gene. The complete list of RNA-Seq results for genes in the TIGR4 adherent sample are listed in Additional file 10.

\section{Adherence characteristics of wild type TIGR4 and isogenic knockout mutants}

On the basis of the microarray results a list of the most highly attractive targets for further investigation was generated (Table 1). Of these, nine regions - 6 individual genes and 3 operons - were selected for knockout experiments (asterisked loci in Table 1, Figure 2, see Methods for prioritization criteria). Mutants were created using insertion-deletion mutagenesis whereby target genes were replaced with a kanamycin resistance-streptomycin sensitive cassette and then incorporated into the genome by genetic transformation and homologous recombination. Three mutants: SP_0783 (vitamin transporter), SP_1270 (alcohol dehydrogenase), and SP_0423 - 


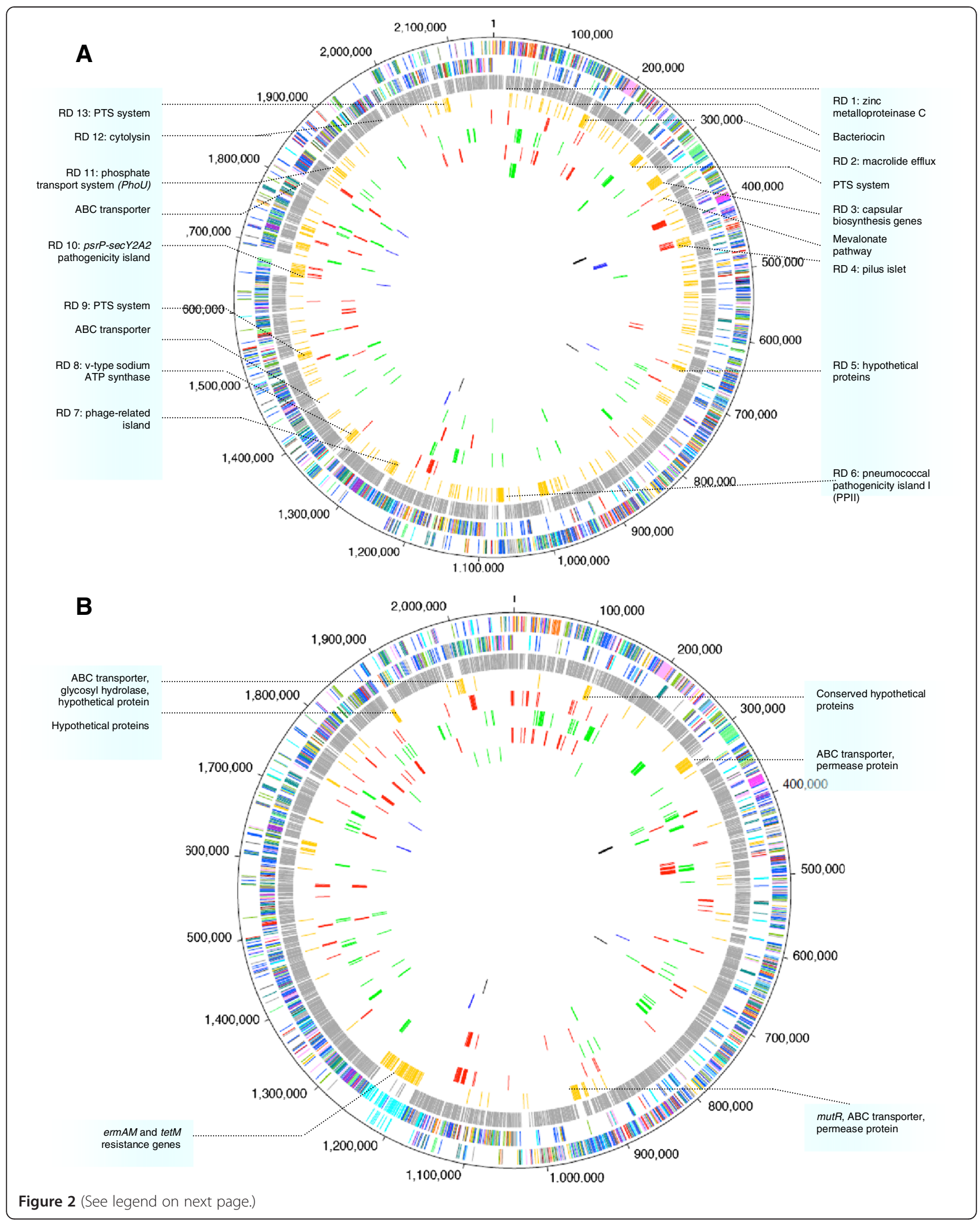


(See figure on previous page.)

Figure 2 Circular representation of the S. pneumoniae TIGR4 (A) and G54 (B) genomes and transcriptomes. The two outermost circles show the predicted coding regions on the plus (outermost) and minus (2nd circle) strands color-coded by functional categories as in Tettelin et al. [60]. The third circle (light grey) shows genes that are shared by both TIGR4 and G54 genomes and the fourth circle (gold) shows strainspecific genes. Colored boxes depict genes in regions of diversity [61] as well as other strain-specific clusters (listed in Additional file 6). The red and green circles correspond to transcriptome results; fifth - genes up-regulated in adherent bacteria; sixth - genes down-regulated in adherent bacteria; seventh - genes up-regulated in non-adherent bacteria; eighth - genes down-regulated in non-adherent bacteria. The two innermost circles correspond to the genes selected for mutagenesis (asterisked loci in Table 1); blue - successfully knocked out (SP_0462-SP_0468, SP_0737, SP_1294-SP_1295, SP_1758, SP_1855 and SP_1922) and black - unsuccessful/not viable (SP_0423-SP_0427, SP_0783 and SP_1270).

SP_0427 (genes encoding the type II fatty acid synthesis pathway), were never obtained despite several attempts at transformation, suggesting that the genes might be essential for survival. The remaining six mutant strains exhibited a reduction in adherence to D562 cells when compared to the wild type strain (range: $30 \%$ to $99.7 \%$ ) (Figure 5). Three of the mutants, the pilus pathogenicity islet (positive control), SP_1855, and SP_1922, were markedly attenuated in adherence. The adherence capacity of two other positive controls - $\triangle s r t A$ and $\triangle p a v A$ was reduced by $60 \%$ and $40 \%$, respectively. The SP_1294, SP_1758 and SP_0737 knockouts showed residual adherence. Growth of the mutants and wild type strain in EMEM were compared (Additional file 5) and the adherence phenotypes were independent of the growth rates of the mutants.

\section{Characterization of SP_1922}

The $\Delta$ SP_1922 mutant was the most thoroughly attenuated in adherence (99.7\%). SP_1922 encodes a hypothetical protein that is present in all pneumococcal genomes published to date. The protein length (238 aa), location, and sequence is identical $(99.9-100 \%)$ in all of the sequenced pneumococcal genomes presented in the Strepneumo Sybil system ([24], see Table 1 for a comparison of the average identity of other genes). By BLASTP analysis (e-value cut-off of $10 \mathrm{e}^{-15}$ ), this gene is conserved in all streptococcal species and is a core gene in several other bacterial species e.g., Moraxella catarrhalis, Escherichia coli, Salmonella enterica, and Shigella sp. Conservation of the gene suggests that it is an important bacterial protein, yet its function is unknown. SP_1922 in S. pneumoniae is located immediately downstream of the pneumolysin gene (ply), which encodes a major pneumococcal toxin that has been implicated in adherence and invasion in several studies [3,25-28]. There are a number of repeat elements (BOX elements and tandem repeats) in the intergenic region between ply and SP_1922. This region appears to be unique to pneumococci. SP_1922 is predicted by the BepiPred software ([29], threshold 2.2) to carry a B-cell epitope. Our microarray results showed that SP_1922 SP_1926 were all up-regulated in TIGR4 upon contact with host cells and down-regulated in the less adherent
G54 strain. Illumina-based RNA sequencing (RNA-Seq, reviewed in [30]) data was generated from TIGR4 adherent samples (36 nt unpaired reads). RNA-Seq coverage revealed that SP_1922 is most likely transcriptionally linked to ply (SP_1923) in a manner consistent with genes organized in an operon (Figure 6, Additional file 10).

To confirm this prediction, the five-gene operon was analyzed with reverse transcriptase PCR (RT-PCR). Using primers spanning various regions within the operon (Additional file 4), RT-PCR showed that hypothetical proteins SP_1922, SP_1924, SP_1925 and SP_1926, were transcribed in an operon with the gene encoding pneumolysin (SP_1923) (Figure 7). Due to paucity of the original TIGR4 adherent RNA samples, the RT-PCR experiments were performed on RNA samples from TIGR4 grown in THYE medium.

\section{Discussion}

Adherence of S. pneumoniae to the human nasopharynx is a critical process and first step that enables the pneumococcus to survive and infect the host. In some cases, the adherent pneumococci penetrate the mucosal epithelium of the nasopharynx and cause systemic disease. The factors that facilitate the transition from adherence to internalization are not well known. We examined the adherence and invasion of pneumococci by using a human pharyngeal epithelial cell line, D562, which is an in vitro model for adherence and for the purpose of this study, mimics the natural site of pneumococcal colonization. Our results show that $S$. pneumoniae TIGR4 strain was more capable of adhering to and being internalized within epithelial cells than G54. This difference was not due to differences in growth patterns, susceptibility to gentamicin, or the G54 strain being more toxic to D562 cells since the EMs showed healthy cells and intact monolayers. Internalization, however, appeared to occur via a similar route in the two strains. Large vacuoles containing pneumococci were observed after infection with either strain. This observation opens up the possibility that pneumococci (or some pneumococcal strains) are not strictly extracellular pathogens as has been traditionally assumed. In support of this finding, Peppoloni et al. showed that the encapsulated strain TIGR4 was protected from 


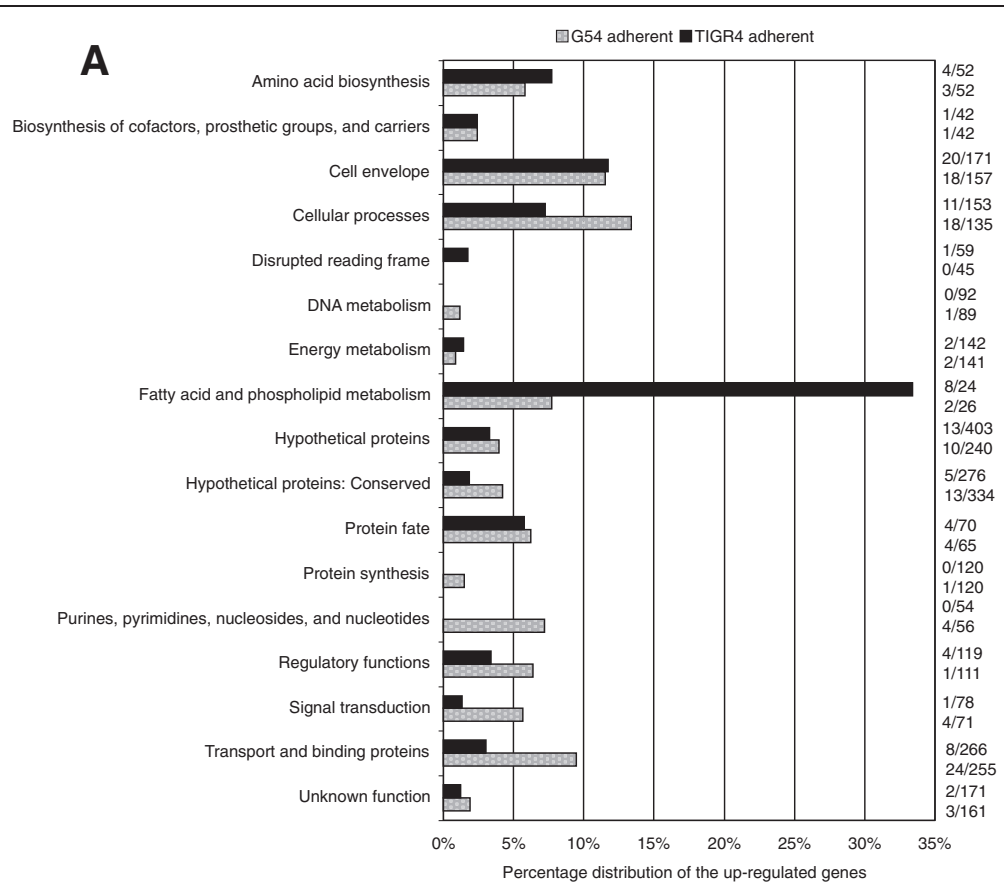

B

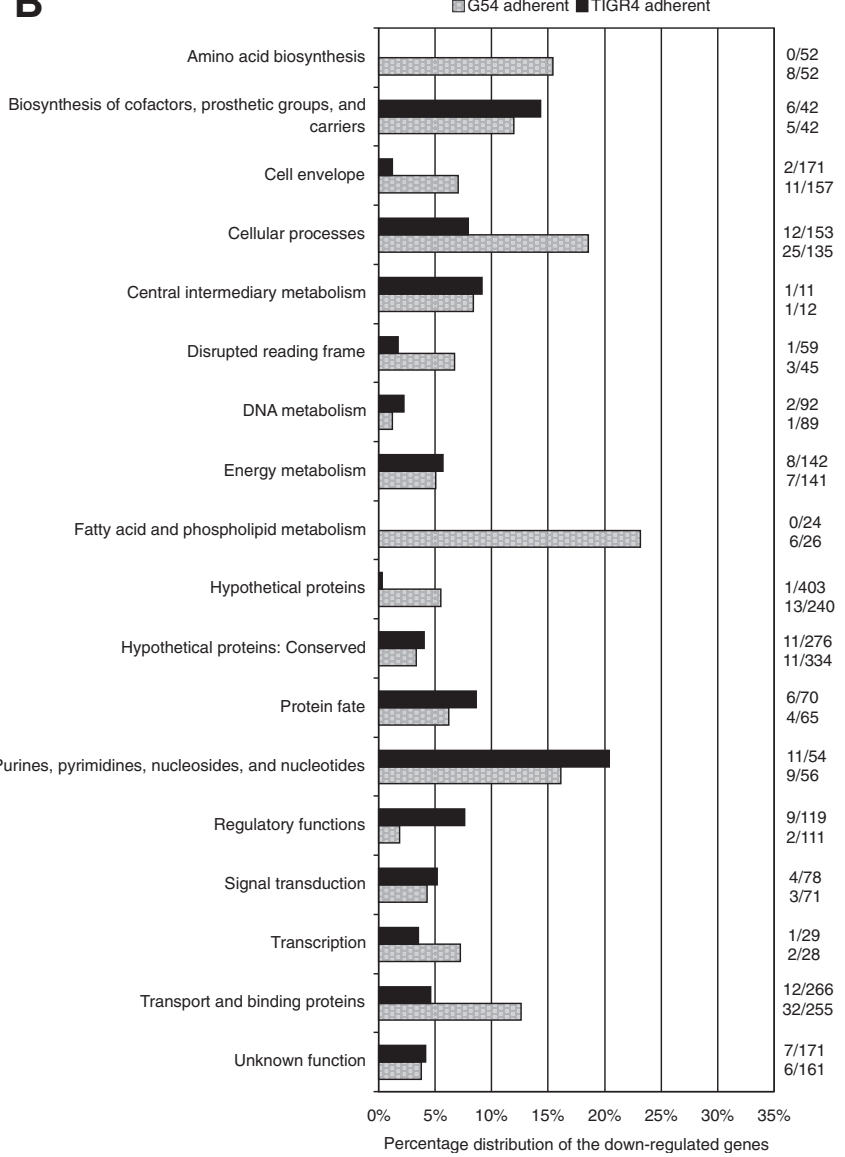

Figure 3 Functional categories of differentially expressed genes in the adherent fraction of TIGR4 and G54. A. Percentage distribution of the up-regulated genes, B. Percentage distribution of the down-regulated genes. TIGR4 genes are depicted as black bars and G54 genes as grey bars. The percentage of genes up- or down-regulated is based on the total number of genes in the genome assigned to a particular category. The numbers on the right show the fractions from where these percentages were derived. 

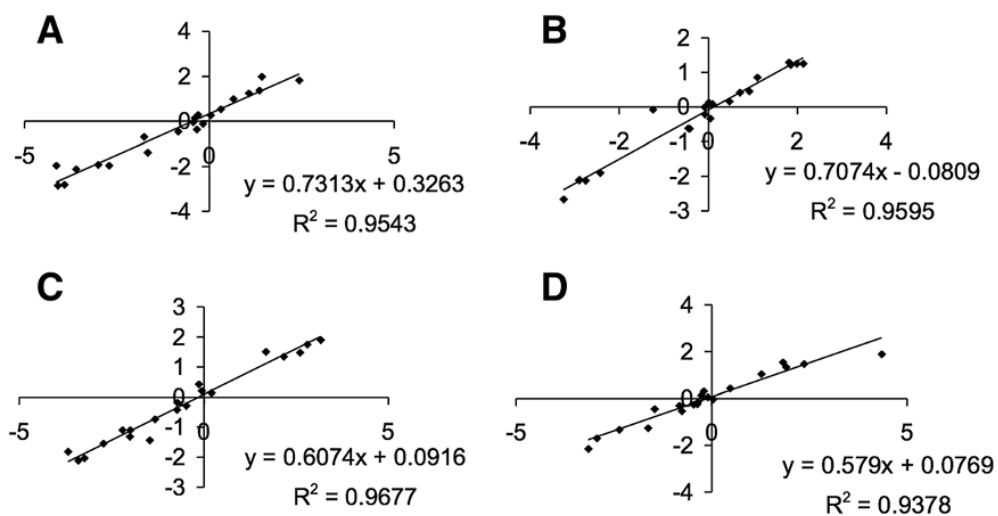

Figure 4 Validation of microarray data by qRT-PCR. mRNA levels of 21 genes obtained by microarray (y-axis) and qRT-PCR ( $x$-axis) are plotted. A, TIGR4 adherent bacteria; B, TIGR4 non-adherent bacteria; C, G54 adherent bacteria; D, G54 non-adherent bacteria. Graphs show a positive correlation between microarray $\log _{2}$ ratios and qRT-PCR $\Delta C \mathrm{Ct}$.

intracellular killing, albeit in a different cell line, murine microgial cell line BV2 [31]. The same observation was made by Briles et al. who found TIGR4 embedded within granulocyte-filled nasal crypts in the sub-mucosa [32]. Rajam et al. [17] demonstrated that the internalization of three pneumococcal strains was in the range of $20-29 \%$, a figure that is comparable to what we found in our study. Our results differ from that of the seminal work by Cundell et al. [33], who showed low invasion capacity of pneumococci. It is worth noting that the invasion assays were performed on a different cell line (primary human umbilical vein endothelial cells vs. D562 cells used in this study), a lower gentamicin concentration $(50 \mu \mathrm{g} / \mathrm{ml}$ vs. $200 \mu \mathrm{g} / \mathrm{ml})$, and the time before gentamicin addition was also significantly shorter (only $30 \mathrm{~min}$ ) perhaps minimizing internalization. The observation that $S$. pneumoniae invasion is cell-type specific has been previously documented [34], with D562 cells exhibiting extensive invasion compared to other cell types.

The phenotypic differences in TIGR4 and G54 can be explained, at least in part, by differences in their genomes and transcriptomes. Genomic differences between the two strains were mainly located in the regions of genomic diversity (RD) (Figure 2). For example, TIGR4, which was superior in adherence and invasion, contained pathogenicity islands $r l r$ and $p s r P-\sec Y 2 A 2$. Previous studies have shown that strains lacking the $r l r$ islet were attenuated in their ability to adhere to eukaryotic cells and to cause invasive disease [35,36] and presence of the psrP-secY2A2 gene cluster has been shown to correlate with the propensity of bacteria to cause invasive pneumococcal disease $[37,38]$. These loci and the others (Additional file 6) may therefore be relevant to TIGR4's interaction with the host but are dispensable in the G54 strain. The strain-specific contribution of certain genes to virulence has been demonstrated in other studies [39-43]. Deletion of cbpA gene from the serotype 4 TIGR4 strain reduced its virulence in a mouse pneumonia model; but had no effect on strains of serotypes 2, 3 and 19F [40]. Similarly, deletion of ppmA (putative proteinase maturation protein A) in a mouse colonization model reduced adherence in TIGR4 and a serotype 35 strain NTC10319 but not in a serotype 2 strain, D39 [39].

Analysis of the transcriptome of TIGR4 and G54 revealed the following biological themes as being the most pronounced:

Genes encoding cellular processes. Genes comA-E, $a d c A-C$, and $a d c R$, involved in inducing competence [44], were all significantly up-regulated in G54 on contact with host cells but only slightly up-regulated in TIGR4. Eleven bacteriocin-associated genes were also regulated in this strain, contributing to the high number of down-regulated genes encoding cellular processes. Bacteriocin-encoding genes have previously been shown to be co-regulated with competence genes in some studies $[45,46]$ but not in others [47]. Pneumolysin (ply) was up-regulated in TIGR4 but down-regulated in G54. The toxin is important for disrupting the tight junctions between epithelial cells and allowing a portal of entry for pneumococcal invasion. Data from Thornton et al. [48] showed that ply induces transcription of host ICAM-1, an epithelial cell adhesion molecule that plays a role in pneumococcal adherence. Ply mutants have been shown to be attenuated in their ability to colonize the nasopharynx in mice [25]. In addition, ply mRNA was shown to be more abundant in the nasopharynx than in blood using two pneumococcal strains, D39 (serotype 2) and WCH16 (serotype 6A) [3,27]. Recent data from Price et al. revealed that Ply is localized to the cell wall in a LytA-independent manner [49]. Given the highly efficient attachment and invasion of epithelial cells by TIGR4 compared to G54 in our experiments, we 
Table 1 List of genes prioritized for functional evaluation of their role in adherence

\begin{tabular}{|c|c|c|c|c|c|c|}
\hline \multicolumn{7}{|c|}{ Fold increase } \\
\hline & & $\begin{array}{l}\text { TIGR4 } \\
\text { adherent/ }\end{array}$ & $\begin{array}{l}\text { G54 } \\
\text { adherent/ }\end{array}$ & $\begin{array}{l}\text { Conserved } \\
{ }^{\S} \text { (Avg. BLASTP Id.) }\end{array}$ & $\begin{array}{l}\text { Predicted surface } \\
\text { exposure" }\end{array}$ & \\
\hline Locus & Annotation & & & & & Reason for selection \\
\hline SP_0018 & Conserved hypothetical protein & 1.7 & 3.3 & yes $(98.4 \%)$ & no & Ratio index $\geq 1.5$ \\
\hline SP_0024 & Hypothetical protein & 2.6 & 0.9 & yes $(98.1 \%)$ & no & Up-regulated in the more adherent strain \\
\hline SP_0025 & Hypothetical protein & 2.7 & 1.1 & yes $(95.7 \%)$ & yes (TmHMM) & Up-regulated in the more adherent strain \\
\hline SP_0026 & Hypothetical protein & 2.2 & $x$ & yes $(78.8 \%)$ & $\begin{array}{l}\text { yes (SP, TmHMM, } \\
\text { AR) }\end{array}$ & Up-regulated in the more adherent strain \\
\hline SP_0099 & Hypothetical protein & 2.2 & 1.2 & yes $(98.7 \%)$ & yes (TmHMM, AR) & Up-regulated in the more adherent strain \\
\hline SP_0100 & Conserved hypothetical protein & 2.2 & 1.4 & yes $(99.3 \%)$ & no & Up-regulated in the more adherent strain \\
\hline SP_0101 & Putative transporter & 1.4 & 1.9 & yes $(99.1 \%)$ & $\begin{array}{l}\text { yes (SP, TmHMM, } \\
\text { AR) }\end{array}$ & Co-opted as part of an operon \\
\hline SP_0415 & Enoyl-CoA hydratase & 1.5 & 0.4 & yes $(99.3 \%)$ & no & Co-opted as part of an operon \\
\hline SP_0416 & Transcriptional regulator, marR family & 1.7 & $x$ & yes $(99.4 \%)$ & no & Co-opted as part of an operon \\
\hline SP_0417 & 3-oxoacyl-(acyl-carrier-protein) synthase III & 1.6 & 1.0 & yes $(99.6 \%)$ & no & Co-opted as part of an operon \\
\hline SP_0418 & Acyl carrier protein & 1.8 & 0.9 & yes $(99.8 \%)$ & no & Ratio index $\geq 1.5$ \\
\hline SP_0419 & Enoyl-(acyl-carrier-protein) reductase & 1.4 & 0.4 & yes $(99.4 \%)$ & no & Co-opted as part of an operon \\
\hline SP_0420 & Malonyl CoA-acyl carrier protein transacylase & 1.9 & 0.6 & yes $(99.5 \%)$ & no & Co-opted as part of an operon \\
\hline SP_0421 & 3-oxoacyl-[acyl-carrier protein] reductase & 2.7 & 0.7 & yes $(99.4 \%)$ & yes (SP) & Up-regulated in the more adherent strain \& Ratio index $\geq 1.5$ \\
\hline SP_0422 & 3-oxoacyl-(acyl-carrier-protein) synthase II & 2.4 & 0.7 & yes $(99.8 \%)$ & yes (SP) & Up-regulated in the more adherent strain \\
\hline SP_0423* & Acetyl-CoA carboxylase, bitoin carboxyl carrier protein & 3.6 & 0.9 & yes $(99.2 \%)$ & no & Up-regulated in the more adherent strain \\
\hline SP_0424* & $\begin{array}{l}\text { Similar to hydroxymyristoyl-(acyl carrier protein) } \\
\text { dehydratase }\end{array}$ & 3.6 & 1.0 & yes $(99.3 \%)$ & no & Up-regulated in the more adherent strain \& Ratio index $\geq 1.5$ \\
\hline SP_0425* & Acetyl-CoA carboxylase, biotin carboxylase & 4.2 & 1.1 & yes $(99.9 \%)$ & no & Up-regulated in the more adherent strain \& Ratio index $\geq 1.5$ \\
\hline SP_0426* & $\begin{array}{l}\text { Acetyl-CoA carboxylase, carboxyl transferase subunit } \\
\text { beta }\end{array}$ & 5.5 & 1.4 & yes $(99.9 \%)$ & no & Up-regulated in the more adherent strain \& Ratio index $\geq 1.5$ \\
\hline SP_0427* & $\begin{array}{l}\text { Acetyl-CoA carboxylase, carboxyl transferase subunit } \\
\text { alpha }\end{array}$ & 2.5 & 1.6 & yes $(99.9 \%)$ & no & Up-regulated in the more adherent strain \& Ratio index $\geq 1.5$ \\
\hline SP_0462* & Transcriptional regulator, putative & 2.1 & NA & no $(90.5 \%)$ & $\begin{array}{l}\text { yes (SP, TmHMM, } \\
\text { BCE) }\end{array}$ & Up-regulated in the more adherent strain \& Ratio index $\geq 1.5$ \\
\hline SP_0463* & Cell wall surface anchor family protein & 2.6 & NA & no $(71.3 \%)$ & $\begin{array}{l}\text { yes (SP, TmHMM, } \\
\text { AR, LPxTG) }\end{array}$ & Up-regulated in the more adherent strain \& Ratio index $\geq 1.5$ \\
\hline SP_0464* & Cell wall surface anchor family protein & 1.9 & NA & no $(98.3 \%)$ & $\begin{array}{l}\text { yes (SP, TmHMM, } \\
\text { AR, LPXTG) }\end{array}$ & Ratio index $\geq 1.5$ \\
\hline SP_0465* & Cell wall surface anchor family protein & 2.1 & NA & no & no & Up-regulated in the more adherent strain \& Ratio index $\geq 1.5$ \\
\hline SP_0466* & Sortase, putative & 2.0 & NA & no $(98.6 \%)$ & yes (TmHMM) & Up-regulated in the more adherent strain \& Ratio index $\geq 1.5$ \\
\hline
\end{tabular}


Table 1 List of genes prioritized for functional evaluation of their role in adherence (Continued)

\begin{tabular}{|c|c|c|c|c|c|c|}
\hline SP_0467* & Sortase, putative & 2.3 & NA & no $(96.0 \%)$ & yes (TmHMM) & Up-regulated in the more adherent strain \& Ratio index $\geq 1.5$ \\
\hline SP_0468* & Sortase, putative & 2.2 & NA & no $(95.8 \%)$ & $\begin{array}{l}\text { yes (SP, TmHMM, } \\
\text { AR, LAS) }\end{array}$ & Up-regulated in the more adherent strain \& Ratio index $\geq 1.5$ \\
\hline SP_0617 & Conserved domain protein & 1.5 & 3.6 & yes $(97.8 \%)$ & yes (SP, TmHMM) & Ratio index $\geq 1.5$ \\
\hline SP_0737* & Sodium-dependent transporter & 2.4 & $x$ & yes $(96.3 \%)$ & yes (SP, TmHMM) & Up-regulated in the more adherent strain \& Ratio index $\geq 1.5$ \\
\hline SP_0738* & Conserved domain protein & 2.0 & $x$ & yes $(99.2 \%)$ & no & Up-regulated in the more adherent strain \& Ratio index $\geq 1.5$ \\
\hline SP_0783* & Conserved hypothetical protein & 1.5 & 1.6 & yes $(96.3 \%)$ & $\begin{array}{l}\text { yes (SP, TmHMM, } \\
\text { AR) }\end{array}$ & Ratio index $\geq 1.5$ \\
\hline SP_0879 & Hypothetical protein & 2 & 3.9 & yes (99.2\%) & yes $(S P, B C E, A R)$ & Up-regulated in both strains \& Ratio index $\geq 1.5$ \\
\hline SP_1003 & Conserved domain protein & 0.8 & 3.9 & yes $(73.3 \%)$ & yes (SP, BCE, AR) & $\begin{array}{l}\text { Up-regulated in the less adherent strain and downregulated } \\
\text { in more adherent strain }\end{array}$ \\
\hline SP_1127 & Hypothetical protein & 1.4 & 1.6 & yes $(98.6 \%)$ & no & Ratio index $\geq 1.5$ \\
\hline SP_1256 & Conserved hypothetical protein & $x$ & 1.9 & yes (96.7\%) & yes (TmHMM) & Ratio index $\geq 1.5$ \\
\hline SP_1270* & Alcohol dehydrogenase, zinc containing & 3.1 & 3.6 & yes $(99.1 \%)$ & no & Up-regulated in both strains \\
\hline SP_1294* & $\begin{array}{l}\text { Crcb protein downstream of a putative chorismate } \\
\text { mutase }\end{array}$ & 2.0 & 1.1 & yes $(98.6 \%)$ & yes (SP, TmHMM) & Up-regulated in the more adherent strain \\
\hline SP_1295* & $\begin{array}{l}\text { Crcb protein downstream of a putative chorismate } \\
\text { mutase }\end{array}$ & 1.8 & 1.1 & yes $(98.1 \%)$ & yes (TmHMM) & Co-opted as part of an operon \\
\hline SP_1600 & Putative membrane protein & 2.0 & 2.0 & yes (99.2\%) & yes (SP, TmHMM) & Up-regulated in both strains \\
\hline SP_1601 & Conserved hypothetical protein & 2.0 & 2.0 & yes $(99.0 \%)$ & yes (SP, TmHMM) & Up-regulated in both strains \& Ratio index $\geq 1.5$ \\
\hline SP_1602 & $\begin{array}{l}\text { Required for expression of the phosphonate } \\
\text { utilization phenotype in } E \text {. coli }\end{array}$ & 2.5 & 2.1 & yes (99.0\%) & no & Up-regulated in both strains \& Ratio index $\geq 1.5$ \\
\hline SP_1758* & $\begin{array}{l}\text { Glycosyl transferases in the psrP-secY2A2 } \\
\text { pathogenicity island }\end{array}$ & 2.2 & $x$ & no (86.3\%) & no & Up-regulated in the more adherent strain \\
\hline SP_1855* & Dehydrogenase & 6.0 & 1.0 & yes (99.7\%) & no & Up-regulated in the more adherent strain \\
\hline SP_1856 & Transcriptional regulator, merR family & 7.6 & 1.1 & yes (99.7\%) & no & Up-regulated in the more adherent strain \\
\hline SP_1857 & Cation efflux system protein & 14.4 & $x$ & yes (98.1\%) & yes (SP, TmHMM) & Up-regulated in the more adherent strain \\
\hline SP_1922* & Conserved hypothetical protein & 2.3 & 1.0 & yes (99.8\%) & yes (BCE) & Up-regulated in the more adherent strain \& Ratio index $\geq 1.5$ \\
\hline SP_1923 & Pneumolysin & 3.0 & 0.5 & yes $(99.8 \%)$ & no & Up-regulated in the more adherent strain \\
\hline SP_1924 & Hypothetical protein & 2.6 & 0.4 & yes $(99.0 \%)$ & no & Up-regulated in the more adherent strain \\
\hline SP_1925 & Hypothetical protein & 2.3 & 0.4 & Yes (98.2) & no & Up-regulated in the more adherent strain \\
\hline
\end{tabular}

* Loci selected for knockout experiments, either individually or as an entire operon.

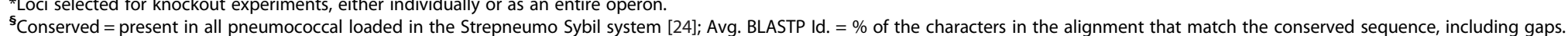

"SP = signal peptide; TmHMM = trans-membrane spans; BCE = B-cell epitopes from BepiPred; AR = antigenic regions from EMBOSS antigenic; LPxTG = proteins covalently linked to peptidoglycan; LAS = lipoprotein attachment site. 


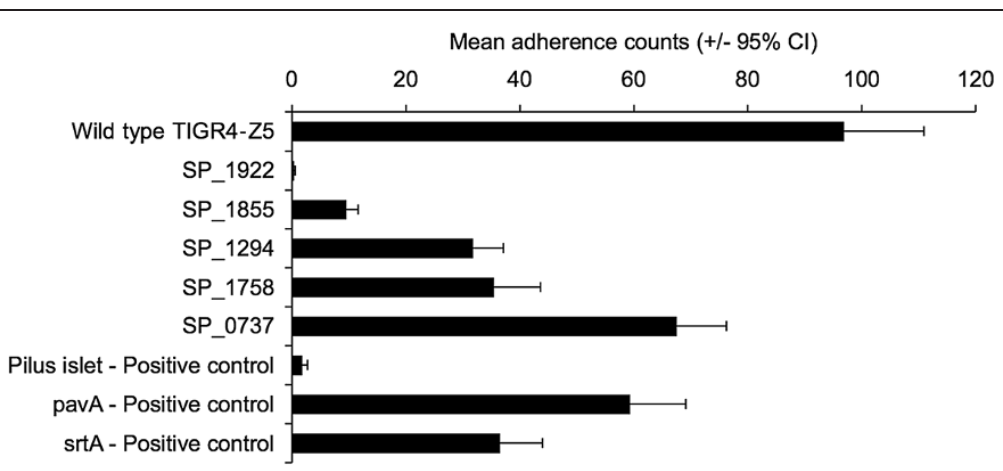

Figure 5 Adherence of knockout mutants compared to the wild type strain. The wild type and mutant strains were incubated for 2 hours with D562 cells and the number of adherent bacteria was determined. The adherence of mutants is given as mean adherence counts from 12 replicate wells (x-axis). Error bars are $95 \%$ confidence intervals observed among the replicates. All means differ significantly from the wild type at $p<0.001$.

speculate that the strain-specific expression of ply might be partly responsible for the observed phenotypic differences, hence supporting the role of pneumolysin in adherence and invasion. The unique up-regulation of ply in TIGR4 and the preferential up-regulation of quorum sensing systems in G54 suggest two different strategies for colonization in the nasopharynx that are consistent with the surface changes depicted by the electron micrographs (Figure 1). An alternative interpretation, which cannot be differentiated in these experiments, is that there are no strain-specific differences but that the observations made represent different stages of cellular infection. If this is the case, the progression through stages appears more rapid for TIGR4.

Interestingly, four hypothetical genes located immediately upstream (SP_1924, SP_1925, SP_1926) and downstream (SP_1922) of the ply gene were expressed in exactly the same manner as ply in both strains suggesting that they might be transcriptionally linked. We performed RT-PCR experiments to confirm expression of SP_1922-SP_1926 as a single operon (Figure 7). Further, Yadav et al. [50] have shown that SP_1924 and

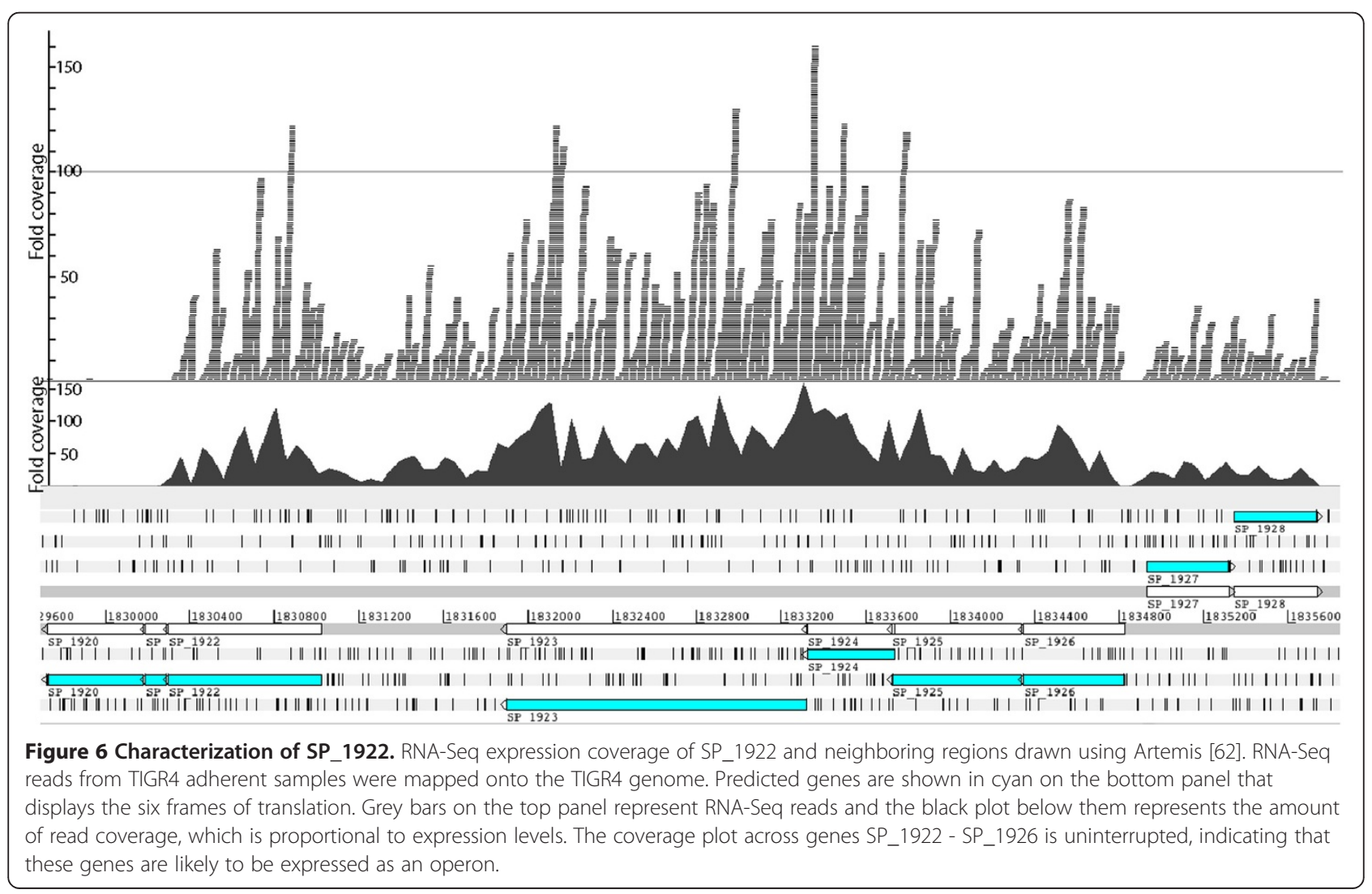




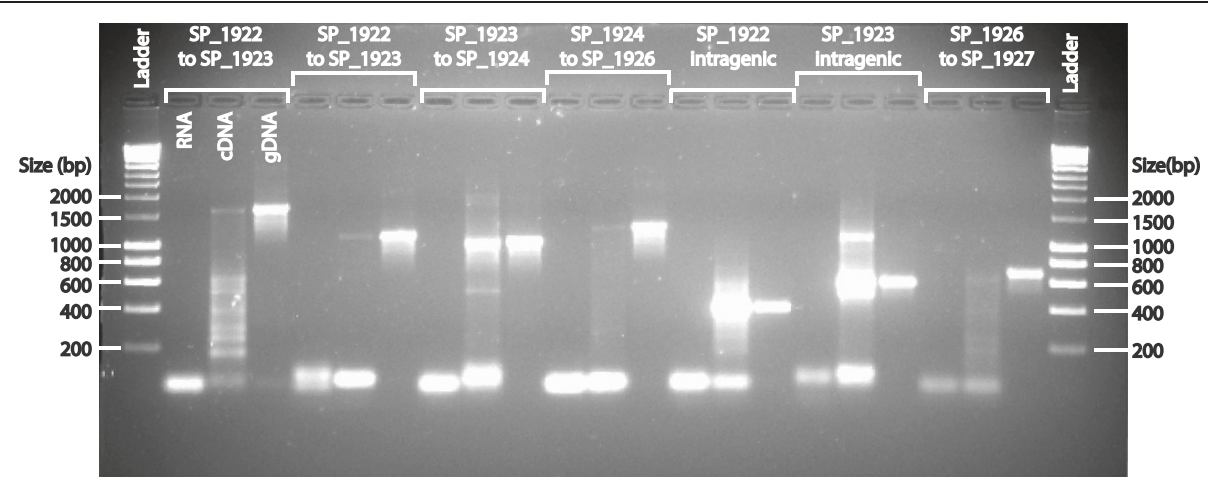

Figure 7 DNA fragments derived from various amplicons of the region spanning SP_1922 - SP_1926. RT-PCR on S. pneumoniae strain TIGR4 grown in rich medium (THYE) showed amplification products of the expected sizes. Lane $1=$ Hyperladder I (Bioline). The remaining lanes represent pairs of primers tested on (i) unprocessed RNA (negative control, confirms absence of contaminating gDNA), cDNA (query), and gDNA (positive control), all from strain TIGR4. Primer pairs spanned: SP_1922 and SP_1923 (ply) (lanes 2-4 \& 5-7), SP_1923 and SP_1924 (lanes 8-10), SP_1924 and SP_1926 (lanes 11-13), SP_1922 intragenic region (lanes 14-16), SP_1923 intragenic region (lanes 17-19), and SP_1926 and SP_1927 (negative control, lanes 20-22). It should be noted that the band of the highest molecular weight in the cDNA lane of the negative control (lane 21) is smaller than the band in the gDNA lane (22) and is part of a non-specific smear of amplification.

SP_1925 are implicated in the transport of Ply from the cytosol to the cell wall compartment, supporting the likelihood of their co-expression. The four hypothetical genes in the ply operon represent interesting candidates for further study to determine their exact role in adherence. Whether or not the BOX elements around this region control the expression of this operon is also a matter worth exploring in the future.

Genes belonging to the cell envelope category. There was enhanced expression of the lic operon in both strains as has been shown by Orihuela et al. [4], suggesting that bacteria were increasing phosphorylcholine levels on their cell wall, thereby enhancing adhesive interaction with host cells. TIGR4 up-regulated the $r l r$ pathogenicity islet genes encoding pneumococcal pili, as well as the glycosylated proteins within RD10 (Additional file 6). Genes in these regions have previously been shown to have adherence functions $[11,35,36,51]$. There were considerable differences in expression in sub-populations of bacteria within the same strain; pili, lic and $p s r P-s e c Y 2 A 2$ operons were more highly expressed in the adherent sub-population as compared to the non-adherent sub-population, when in the presence of host cells.

Genes encoding transport and binding proteins. Genes belonging to the $p s a$ operon, a manganese $\mathrm{ABC}$ transporter system, were significantly up-regulated in both strains. These genes have previously been implicated in pneumococcal pathogenesis, specifically adhesion $[4,6,8,52,53]$. phnA, encoding an alkylphosphonate utilization protein transporter, as well as a gene encoding a putative membrane transporter (SP_1600), and a hypothetical gene (SP_1601) downstream of it were up-regulated in both strains. In E. coli, the phn operon consists of 14 genes that are regulated by the phosphate (pho) system [54]. Induction of phn genes in our study suggests that overcoming phosphate limitation might be important for adherence and invasion. Interestingly, $a d h C$ (SP_1855), SP_1856, and $c z c D$ (SP_1857) encoding a zinc-containing alcohol dehydrogenase, a MerR family regulator, and a zinc efflux pump, respectively, were significantly up-regulated in TIGR4. AdhC and the MerR family regulator have previously been shown to be important for virulence [55]. In a separate study [56], these genes appeared to be required for resistance to nitric oxide stress, but did not play a key role in nasopharyngeal colonization in vivo. Nitric oxide is recognized as a key component of the innate immune response. Nitric oxide can inhibit bacterial DNA replication via the release of zinc from metalloproteins and can also hinder bacterial respiration. Hence bacteria e.g. pneumococci, Haemophilus influenzae and $E$. coli have developed nitric oxide resistance mechanisms such as the $a d h C-c z c D$ system [56-59]. Expression of these genes in our study is consistent with our earlier observation that a sub-population of bacteria penetrated the epithelial cells; the observed regulation might be an adaptation to the intracellular environment. It is conceivable that induction of ply by TIGR4 resulted in epithelial damage, which in turn led to triggering of nitric oxide production by the host cells, placing bacteria under nitrosative stress. AdhC (SP_1855) is up-regulated to reduce the nitric oxide, therefore allowing internalized bacteria to resist killing.

Other regulated genes. Genes encoding metabolismassociated proteins were differentially expressed upon adherence of pneumococci to D562 cells. These include genes involved in amino acid biosynthesis, nucleotide, sugar, fatty acid and phospholipids metabolism. A number of the differentially regulated genes encoded hypothetical proteins or proteins with unknown functions. Many of these proteins are predicted to be $>100$ aa (low probability to arise from randomly occurring, spurious 
open reading frames) and have signal peptides or other motifs indicating that they are likely to be functional surface localized proteins (Table 1). These properties make them good candidates for further characterization of their utility as vaccine antigens.

Our mutagenesis data showed that deletion of both SP_1922 (encoding a conserved hypothetical protein) and the $r l r$ islet, independently resulted in almost complete attenuation of adherence. Though we expected the $r l r$ mutant to be attenuated in adherence, the extent to which adherence was reduced (98\%) was surprising since it is known that strains without pili bind to epithelial cells, albeit less efficiently [35]. It is conceivable that SP_1922 and rlr might act in synergy or might be coregulated and therefore deletion of one gene affects the function of the other. It has previously been shown that adherence via pili is coordinated with adherence by other adhesins [36]. Deletion of the whole pilus islet in our study might have affected the regulation of some genes (coding for adhesins) outside the islet, SP_1922 included. On the other hand, deletion of SP_1922 totally abolishes all the adhesion seen in this in vitro model. Of note, the knockout was carefully constructed to prevent interfering with ply, which is upstream of this gene. In addition, the activity of pneumolysin in the SP_1922 mutant was confirmed to be similar to that of the wild type using a hemolytic activity assay (Additional file 11). We are tempted to speculate that this gene encodes the most important adhesin in this in vitro assay system. Deletion of this gene probably changes an important adhesive property without which other adhesins cannot function. Further investigations are needed to elucidate our findings.

\section{Conclusion}

We have identified a novel role for several pneumococcal genes in adherence to pharyngeal epithelial cells. This work therefore identifies a short list of genes that encode proteins that can be further evaluated as potential antimicrobial targets or non-capsular vaccine candidates if immunogenicity can be demonstrated in future studies.

\section{Additional files}

Additional file 1: Is a figure showing an overview of the microarray experimental set up.

Additional file 2: Is a table listing the primers used for qRT-PCR analysis of bacterial genes.

Additional file 3: Is a table listing the primers used for knockout mutagenesis.

Additional file 4: Is a table listing the primers used for RT-PCR confirmation of the pneumolysin operon.
Additional file 5: Shows growth curves in EMEM of G54 and TIGR4 wild type strains, as well as the TIGR4 isogenic mutants.

Additional file 6: Is a table listing the regions of diversity between TIGR4 and G54 strain.

Additional file 7: Is a table listing genes differentially expressed in cell-adherent pneumococci vs. culture medium control pneumococci.

Additional file 8: Is a table listing genes differentially expressed in cell-adherent pneumococci vs. non-adherent pneumococci exposed to D562 pharyngeal cells.

Additional file 9: Is a figure showing the correlation between RNASeq average coverage and qRT-PCR thresholds.

Additional file 10: Is a table showing the complete list of RNA-Seq results.

Additional file 11: Is a table showing the hemolytic activity of pneumolysin in TIGR4 wild type and its SP_1922 isogenic mutant strain.

\section{Abbreviations}

D562 cells: Detroit 562 cells; RNA-Seq: RNA sequencing; Reverse transcriptase PCR: RT-PCR; SAM: Significance analysis of microarrays; Ply: Pneumolysin; RD: Regions of genomic diversity; PsaA: Pneumococcal surface adhesion A; PsrP: Pneumococcal serine repeat protein; CbpA: Choline binding protein A.

\section{Competing interests}

JAGS reports receiving a grant from GlaxoSmithKline Biologicals (Anthony Scott, Kayla Laserson; $\$ 2,575,975$; Oct 2010-Sep 2013) for a study entitled: 'A phase IV multi-site observational epidemiology study to assess potential risk for adverse events following immunization that may be associated with misuse of a two-dose vial of 10-valent Pneumococcal Conjugate Vaccine (Synflorix) in Kenya'.

\section{Authors' contributions}

All authors read and approved the final manuscript. SZKM carried out the adherence and invasion assays, prepared cells for electron microscopy, performed the pneumococcal microarray experiments and knockout experiments, analyzed the data, and drafted the manuscript. SRS participated in the design and performance of bacterial adherence and invasion assays, electron microscopy, RNA extractions and drafting of the manuscript. JCDH participated in the design and analysis of all experiments involving molecular work in this manuscript. NK performed the PCR for confirmation of operon structure and GRT-PCR experiments. NI participated in the pneumococcal microarray experiments. DRR participated in the bioinformatics analysis of microarray work. UF, THC, LT, XL participated in the RNA sequencing. CSG performed the electron microscopy. JS and GMC participated in the design of the study. SKH participated in the design and performance of knockout experiments. JAGS and HT conceived the study, participated in the study design and development, analysis, and drafting of the manuscript.

\section{Acknowledgements}

This work was supported by grants from the Wellcome Trust to Prof. Anthony Scott (081835) and University of Maryland internal funds to Dr. Hervé Tettelin. Sheila Z. Kimaro Mlacha was supported by strategic training award No. 084538 to the KEMRI-Wellcome Trust Research Programme. This paper is published with the permission of the Director, Kenya Medical Research Institute. The authors wish to thank the Pathogen Functional Genomics Resource Center (PFGRC) at The Institute for Genomic Research (TIGR) / J. Craig Venter Institute (JCVI) for providing microarray slides, Shankar Rajam, Yulanda Williamson (Centers for Disease Control and Prevention, CDC) and Pat Cohen (University of Alabama, Birmingham) for technical assistance, and Margaret Mackinnon and Greg Fegan (KEMRI/Wellcome Trust) for expert advice on statistical methods.

\section{Author details}

'Kenya Medical Research Institute, Wellcome Trust Research Programme, Kilifi, Kenya. ${ }^{2}$ Department of Microbiology and Immunology, Institute for Genome Sciences, University of Maryland School of Medicine, 801 W. 
Baltimore Street, Baltimore, MD 21201, USA. ${ }^{3}$ Division of Bacterial Diseases, Centers for Disease Control and Prevention, Atlanta, GA, USA. ${ }^{4}$ Division of High-Consequence Pathogens and Pathology, Centers for Disease Control and Prevention, Atlanta, GA, USA. ${ }^{5}$ Department of Microbiology, University of Alabama at Birmingham, Birmingham, AL, USA. ${ }^{6}$ Nuffield Department of Clinical Medicine, University of Oxford, Oxford, UK. 'Present address: Respiratory \& Meningeal Pathogens Research Unit, University of the Witwatersrand/Medical Research Council, Johannesburg, South Africa.

Received: 10 October 2012 Accepted: 24 May 2013

Published: 9 June 2013

\section{References}

1. O'Brien KL, Wolfson L, Watt JP, Henkle E, Deloria-Knoll M, McCall N, Lee E, Mulholland K, Levine OS, Cherian T: Burden of disease caused by Streptococcus pneumoniae in children younger than 5 years: global estimates. Lancet 2009, 374:893-902.

2. Roush SW, Murphy TV: Historical comparisons of morbidity and mortality for vaccine-preventable diseases in the United States. JAMA 2007, 298:2155-2163.

3. LeMessurier KS, Ogunniyi AD, Paton JC: Differential expression of key pneumococcal virulence genes in vivo. Microbiology 2006, 152:305-311.

4. Orihuela CJ, Radin JN, Sublett JE, Gao G, Kaushal D, Tuomanen El: Microarray analysis of pneumococcal gene expression during invasive disease. Infect Immun 2004, 72:5582-5596.

5. Song XM, Connor W, Hokamp K, Babiuk LA, Potter AA: Streptococcus pneumoniae early response genes to human lung epithelial cells. $B M C$ Res Notes 2008, 1:64.

6. Song XM, Connor W, Hokamp K, Babiuk LA, Potter AA: Transcriptome studies on Streptococcus pneumoniae, illustration of early response genes to THP-1 human macrophages. Genomics 2009, 93:72-82.

7. Williamson YM, Gowrisankar R, Longo DL, Facklam R, Gipson IK, Ades EP, Carlone GM, Sampson JS: Adherence of nontypeable Streptococcus pneumoniae to human conjunctival epithelial cells. Microb Pathog 2008, 44:175-185.

8. Romero-Steiner S, Pilishvili T, Sampson JS, Johnson SE, Stinson A, Carlone GM, Ades EW: Inhibition of pneumococcal adherence to human nasopharyngeal epithelial cells by anti-PsaA antibodies. Clin Diagn Lab Immunol 2003, 10:246-251.

9. Orihuela CJ: Role played by psrP-sec Y2A2 (accessory region 34 ) in the invasive disease potential of Streptococcus pneumoniae. J Infect Dis 2009, 200:1180-1181. author reply 1181-1182.

10. Orihuela CJ, Mahdavi J, Thornton J, Mann B, Wooldridge KG, Abouseada N, Oldfield NJ, Self T, Ala'Aldeen DA, Tuomanen El: Laminin receptor initiates bacterial contact with the blood brain barrier in experimental meningitis models. J Clin Invest 2009, 119:1638-1646.

11. Nelson AL, Ries J, Bagnoli F, Dahlberg S, Falker S, Rounioja S, Tschop J, Morfeldt E, Ferlenghi I, Hilleringmann M, et al: RrgA is a pilus-associated adhesin in Streptococcus pneumoniae. Mol Microbiol 2007, 66:329-340.

12. Pracht D, Elm C, Gerber J, Bergmann S, Rohde M, Seiler M, Kim KS, Jenkinson HF, Nau R, Hammerschmidt S: PavA of Streptococcus pneumoniae modulates adherence, invasion, and meningeal inflammation. Infect Immun 2005, 73:2680-2689.

13. Hilleringmann M, Giusti F, Baudner BC, Masignani V, Covacci A, Rappuoli R, Barocchi MA, Ferlenghi I: Pneumococcal pili are composed of protofilaments exposing adhesive clusters of Rrg A. PLoS Pathog 2008, 4:e1000026.

14. Papasergi S, Garibaldi M, Tuscano G, Signorino G, Ricci S, Peppoloni S, Pernice I, Lo Passo C, Teti G, Felici F, et al: Plasminogen- and fibronectinbinding protein B is involved in the adherence of Streptococcus pneumoniae to human epithelial cells. J Biol Chem 2010, 285:7517-7524.

15. Yamaguchi M, Terao Y, Mori Y, Hamada S, Kawabata S: PfbA, a novel plasmin- and fibronectin-binding protein of Streptococcus pneumoniae, contributes to fibronectin-dependent adhesion and antiphagocytosis. J Biol Chem 2008, 283:36272-36279.

16. Weiser JN, Austrian R, Sreenivasan PK, Masure HR: Phase variation in pneumococcal opacity: relationship between colonial morphology and nasopharyngeal colonization. Infect Immun 1994, 62:2582-2589.

17. Rajam G, Phillips DJ, White E, Anderton J, Hooper CW, Sampson JS, Carlone GM, Ades EW, Romero-Steiner S: A functional epitope of the pneumococcal surface adhesin A activates nasopharyngeal cells and increases bacterial internalization. Microb Pathog 2008, 44:186-196.

18. Mollenhauer HH: Plastic Embedding Mixtures for Use in Electron Microscopy. Stain Technol 1964, 39:111-114.

19. Jiang SM, Ishmael N, Dunning Hotopp J, Puliti M, Tissi L, Kumar N, Cieslewicz MJ, Tettelin H, Wessels MR: Variation in the group B Streptococcus CsrRS regulon and effects on pathogenicity. J Bacteriol 2008, 190:1956-1965.

20. Saeed Al, Sharov V, White J, Li J, Liang W, Bhagabati N, Braisted J, Klapa M, Currier T, Thiagarajan M, et al: TM4: a free, open-source system for microarray data management and analysis. Biotechniques 2003, 34:374-378.

21. Livak KJ, Schmittgen TD: Analysis of relative gene expression data using real-time quantitative PCR and the 2(-Delta Delta C(T)) Method. Methods 2001, 25:402-408.

22. Lau PC, Sung CK, Lee JH, Morrison DA, Cvitkovitch DG: PCR ligation mutagenesis in transformable streptococci: application and efficiency. J Microbiol Methods 2002, 49:193-205.

23. Sung CK, Li H, Claverys JP, Morrison DA: An rpsL cassette, janus, for gene replacement through negative selection in Streptococcus pneumoniae. Appl Environ Microbiol 2001, 67:5190-5196.

24. Riley DR, Angiuoli SV, Crabtree J, Dunning Hotopp JC, Tettelin H: Using Sybil for interactive comparative genomics of microbes on the web. Bioinformatics 2012, 28:160-166.

25. Ogunniyi AD, LeMessurier KS, Graham RM, Watt JM, Briles DE, Stroeher UH, Paton JC: Contributions of pneumolysin, pneumococcal surface protein $A$ (PspA), and PspC to pathogenicity of Streptococcus pneumoniae D39 in a mouse model. Infect Immun 2007, 75:1843-1851.

26. Rubins JB, Paddock AH, Charboneau D, Berry AM, Paton JC, Janoff EN: Pneumolysin in pneumococcal adherence and colonization. Microb Pathog 1998, 25:337-342.

27. Mahdi LK, Ogunniyi AD, LeMessurier KS, Paton JC: Pneumococcal virulence gene expression and host cytokine profiles during pathogenesis of invasive disease. Infect Immun 2008, 76:646-657.

28. Rubins JB, Janoff EN: Pneumolysin: a multifunctional pneumococcal virulence factor. J Lab Clin Med 1998, 131:21-27.

29. Larsen JE, Lund O, Nielsen M: Improved method for predicting linear B-cell epitopes. Immunome Res 2006, 2:2

30. Croucher NJ, Thomson NR: Studying bacterial transcriptomes using RNAseq. Curr Opin Microbiol 2010, 13:619-624.

31. Peppoloni S, Ricci S, Orsi CF, Colombari B, De Santi MM, Messino M, Fabio G, Zanardi A, Righi E, Braione V, et al: The encapsulated strain TIGR4 of Streptococcus pneumoniae is phagocytosed but is resistant to intracellular killing by mouse microglia. Microbes Infect 2010, 12:990-1001.

32. Briles DE, Novak L, Hotomi M, Van Ginkel FW, King J: Nasal colonization with Streptococcus pneumoniae includes subpopulations of surface and invasive pneumococci. Infect Immun 2005, 73:6945-6951.

33. Cundell DR, Gerard NP, Gerard C, Idanpaan-Heikkila I, Tuomanen El: Streptococcus pneumoniae anchor to activated human cells by the receptor for platelet-activating factor. Nature 1995, 377:435-438.

34. Brock SC, McGraw PA, Wright PF, Crowe JE Jr: The human polymeric immunoglobulin receptor facilitates invasion of epithelial cells by Streptococcus pneumoniae in a strain-specific and cell type-specific manner. Infect Immun 2002, 70:5091-5095.

35. Barocchi MA, Ries J, Zogaj X, Hemsley C, Albiger B, Kanth A, Dahlberg S, Fernebro J, Moschioni M, Masignani $V$, et al: A pneumococcal pilus influences virulence and host inflammatory responses. Proc Natl Acad Sci USA 2006, 103:2857-2862.

36. Rosch JW, Mann B, Thornton J, Sublett J, Tuomanen E: Convergence of regulatory networks on the pilus locus of Streptococcus pneumoniae. Infect Immun 2008, 76:3187-3196.

37. Obert C, Sublett J, Kaushal D, Hinojosa E, Barton T, Tuomanen El, Orihuela CJ: Identification of a Candidate Streptococcus pneumoniae core genome and regions of diversity correlated with invasive pneumococcal disease. Infect Immun 2006, 74:4766-4777.

38. Munoz-Almagro C, Selva L, Sanchez CJ, Esteva C, De Sevilla MF, Pallares R, Orihuela CJ: PsrP, a protective pneumococcal antigen, is highly prevalent in children with pneumonia and is strongly associated with clonal type. Clin Vaccine Immunol 2010, 17:1672-1678.

39. Cron LE, Bootsma HJ, Noske N, Burghout P, Hammerschmidt S, Hermans PW: Surface-associated lipoprotein PpmA of Streptococcus pneumoniae is 
involved in colonization in a strain-specific manner. Microbiology 2009, 155:2401-2410.

40. Kerr AR, Paterson GK, McCluskey J, lannelli F, Oggioni MR, Pozzi G, Mitchell $\mathrm{TJ}$ : The contribution of PspC to pneumococcal virulence varies between strains and is accomplished by both complement evasion and complement-independent mechanisms. Infect Immun 2006, 74:5319-5324.

41. Yuste J, Khandavilli S, Ansari N, Muttardi K, Ismail L, Hyams C, Weiser J, Mitchell T, Brown JS: The effects of PspC on complement-mediated immunity to Streptococcus pneumoniae vary with strain background and capsular serotype. Infect Immun 2010, 78:283-292.

42. Harvey RM, Stroeher UH, Ogunniyi AD, Smith-Vaughan HC, Leach AJ, Paton $J C$ : A variable region within the genome of Streptococcus pneumoniae contributes to strain-strain variation in virulence. PLoS One 2011, 6:e19650.

43. McAllister LJ, Ogunniyi AD, Stroeher UH, Leach AJ, Paton JC: Contribution of Serotype and Genetic Background to Virulence of Serotype 3 and Serogroup 11 Pneumococcal Isolates. Infect Immun 2011, 70:4839-4849.

44. Claverys JP, Dintilhac A, Mortier-Barriere I, Martin B, Alloing G: Regulation of competence for genetic transformation in Streptococcus pneumoniae. Soc Appl Bacteriol Symp Ser 1997, 26:32S-41S.

45. Peterson SN, Sung CK, Cline R, Desai BV, Snesrud EC, Luo P, Walling J, Li H, Mintz M, Tsegaye G, et al: Identification of competence pheromone responsive genes in Streptococcus pneumoniae by use of DNA microarrays. Mol Microbiol 2004, 51:1051-1070.

46. Fontaine L, Boutry C, Guedon E, Guillot A, Ibrahim M, Grossiord B, Hols P: Quorum-sensing regulation of the production of Blp bacteriocins in Streptococcus thermophilus. J Bacteriol 2007, 189:7195-7205.

47. Claverys JP, Havarstein LS: Extracellular-peptide control of competence for genetic transformation in Streptococcus pneumoniae. Front Biosci 2002 7:d1798-d1814.

48. Thornton J, McDaniel LS: THP-1 monocytes up-regulate intercellular adhesion molecule 1 in response to pneumolysin from Streptococcus pneumoniae. Infect Immun 2005, 73:6493-6498.

49. Price KE, Camilli A: Pneumolysin localizes to the cell wall of Streptococcus pneumoniae. J Bacteriol 2009, 191:2163-2168.

50. Yadav P, Thompson C, Lu J, Malley R: Genetic requirements for pneumolysin localization. Abstractcts 7 th International Symposium on Pneumococci and Pneumococcal Diseases (ISPPD-7). Israel: Tel Aviv; 2010:35.

51. Shivshankar P, Sanchez C, Rose LF, Orihuela CJ: The Streptococcus pneumoniae adhesin PsrP binds to Keratin 10 on lung cells. Mol Microbiol 2009, 73:663-679.

52. Berry AM, Paton JC: Sequence heterogeneity of PsaA, a 37-kilodalton putative adhesin essential for virulence of Streptococcus pneumoniae. Infect Immun 1996, 64:5255-5262.

53. Sampson JS, O'Connor SP, Stinson AR, Tharpe JA, Russell H: Cloning and nucleotide sequence analysis of psaA, the Streptococcus pneumoniae gene encoding a 37-kilodalton protein homologous to previously reported Streptococcus sp. adhesins. Infect Immun 1994, 62:319-324

54. Metcalf WW, Wanner BL: Mutational analysis of an Escherichia coli fourteen-gene operon for phosphonate degradation, using TnphoA' elements. J Bacteriol 1993, 175:3430-3442.

55. Hava DL, Camilli A: Large-scale identification of serotype 4 Streptococcus pneumoniae virulence factors. Mol Microbiol 2002, 45:1389-1406.

56. Stroeher UH, Kidd SP, Stafford SL, Jennings MP, Paton JC, McEwan AG: A pneumococcal MerR-like regulator and S-nitrosoglutathione reductase are required for systemic virulence. J Infect Dis 2007, 196:1820-1826.

57. Kloosterman TG, van der Kooi-Pol MM, Biilsma JJ, Kuipers OP: The novel transcriptional regulator SczA mediates protection against $\mathrm{Zn} 2+$ stress by activation of the $\mathrm{Zn} 2+-$ resistance gene $\mathrm{CzCD}$ in Streptococcus pneumoniae. Mol Microbiol 2007, 65:1049-1063.

58. Kidd SP, Jiang D, Jennings MP, McEwan AG: Glutathione-dependent alcohol dehydrogenase $\mathrm{AdhC}$ is required for defense against nitrosative stress in Haemophilus influenzae. Infect Immun 2007, 75:4506-4513.
59. Liu L, Hausladen A, Zeng M, Que L, Heitman J, Stamler JS: A metabolic enzyme for S-nitrosothiol conserved from bacteria to humans. Nature 2001, 410:490-494.

60. Tettelin H, Nelson KE, Paulsen IT, Eisen JA, Read TD, Peterson S, Heidelbers J, DeBoy RT, Haft DH, Dodson RJ, et al: Complete genome sequence of a virulent isolate of Streptococcus pneumoniae. Science 2001, 293:498-506.

61. Tettelin H, Hollingshead S: Comparative genomics of Streptococcus pneumoniae: intrastrain diversity and genome plasticity. The pneumococcus. Washington, DC: ASM Press; 2004:15-29.

62. Rutherford K, Parkhill J, Crook J, Horsnell T, Rice P, Rajandream MA, Barrell B: Artemis: sequence visualization and annotation. Bioinformatics 2000, 16:944-945

doi:10.1186/1471-2164-14-383

Cite this article as: Kimaro Mlacha et al:: Phenotypic, genomic, and transcriptional characterization of Streptococcus pneumoniae interacting with human pharyngeal cells. BMC Genomics 2013 14:383.

\section{Submit your next manuscript to BioMed Central and take full advantage of:}

- Convenient online submission

- Thorough peer review

- No space constraints or color figure charges

- Immediate publication on acceptance

- Inclusion in PubMed, CAS, Scopus and Google Scholar

- Research which is freely available for redistribution

Submit your manuscript at www.biomedcentral.com/submit
() Biomed Central 